\title{
Associations Among Internet Addiction, Genetic Polymorphisms, Family Functioning, and Psychopathological Risk: Cross-Sectional Exploratory Study
}

Luca Cerniglia $^{1 *}, \mathrm{PhD}$; Silvia Cimino ${ }^{2 *}, \mathrm{PhD}$; Eleonora Marzilli ${ }^{2}, \mathrm{MA}$; Esterina Pascale ${ }^{2}, \mathrm{PhD}$; Renata Tambelli ${ }^{2}$, Prof Dr

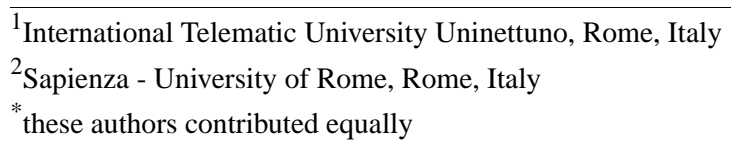

Corresponding Author:

Luca Cerniglia, $\mathrm{PhD}$

International Telematic University Uninettuno

Corso Vittorio Emanuele II, 39

Rome, 00186

Italy

Phone: 3906679201

Email:1.cerniglia@uninettunouniversity.net

\section{Abstract}

Background: International research has emphasized that youths are at higher risk for the onset of internet addiction (IA), but studies investigating biological, psychological, and social factors associated with this condition are limited.

Objective: This study aims to investigate the possible association between IA and genetic polymorphisms in monoamine oxidase A (MAO-A), serotonin-transporter (5-HTTPR), dopamine receptor (DRD4), and dopamine transporter (DAT1) genes by considering the role played by the perception of young adults in their family functioning and their depression, anxiety, and avoidant personality problems.

Methods: In a sample of 104 male and female young adults aged between 19 and 23 years (mean age 21.87, SD 2.29 years) recruited from universities in the central southern part of Italy, we addressed the presence of IA using the Young criteria of the IA test. Moreover, the perception of young adults of their family functioning and their psychopathological symptoms were assessed through the Family Assessment Device (FAD) and the Adult Self-Report, respectively.

Results: We found no significant association between IA and any genetic polymorphisms, neither among males or females. Young adults with IA reported significantly higher scores in the subscale of FAD affective responsiveness (AR; $P=.01)$ and in depressive problems $(P=.02)$, anxiety problems $(P=.009)$, and avoidant personality problems $(P=.003)$ than those in the control group. Results of mediation analyses showed a mediation role played by depressive symptoms $(B=0.99 ; 95 \%$ CI 0.22 to 1.97$)$ and avoidant personality problems $(B=1.09 ; 95 \% \mathrm{CI} 0.32$ to 2.05$)$ of young adults on the relationship between the FAD, AR, and IA. Finally, this relationship was moderated by the genotype of the 5-HTTLPR $(P<.001)$, DAT1 $(P<.001)$, and MAO-A $(P<.001)$ genes in young adults.

Conclusions: This exploratory study supports the recent evidence on the mutual relationship among biological, individual, and social risk factors associated with IA in young adulthood. Our findings may have important clinical implications for the development of prevention and treatment programs.

(JMIR Ment Health 2020;7(12):e17341) doi: 10.2196/17341

\section{KEYWORDS}

internet addiction; mobile phones; family functioning; depression; anxiety; avoidant personality; MAO-A; 5-HTTPR; DRD4; DAT1 


\section{Introduction}

\section{Background}

Over the past decade, the diffusion and use of the internet has grown rapidly, especially among adolescents and young adults [1]. From a developmental perspective, young adults may face important challenges in relationships with their family, peers, and society, the outcomes of which can be mediated and moderated by their psychological and biological characteristics [2]. On the one hand, youths can benefit from the digital revolution, which allows them to instantly search for information and communicate with others around the world [3]. In addition, there is evidence that mobile health smartphone apps can prevent diseases and improve the quality of life in young people [4]. However, on the other hand, scientific literature indicates that young adults are at higher risk of using the internet in a maladaptive way [5,6], to the point of developing internet addiction (IA) symptoms [7]. However, to date, official diagnostic criteria have not been identified [8]; although, the revision of the fifth edition of the Diagnostic and Statistical Manual of Mental Disorders (DSM-5) [9] has included an internet-related condition-the internet gaming disorder in Section III. This condition has been defined as a behavioral addiction that eventually leads to loss of control over internet gaming and functional impairment [10]. Some authors have defined IA as uncontrollable and obsessive-compulsive use of the internet, preoccupations regarding computer or technological devices, inability to control their use, poor time management, craving, and interpersonal problems [11-14]. Epidemiological studies have shown a prevalence of IA ranging from $6 \%$ to $35 \%$ among young adults $[15,16]$ and from $6 \%$ to $21 \%$ among adolescents [17]. Although the highest prevalence has been reported among males [18], recent evidence has underlined an increase among females, with no significant gender difference [19]. The Developmental Psychopathology theoretical framework [20] offers a valid model to conceptualize clinical and subclinical psychological difficulties in young adulthood (such as IA) as it considers the development as a result of mutual influences between individual inherited genetic vulnerabilities and the quality of social experiences [21,22], particularly within the family context [23].

\section{Genetic Influences on IA}

Genes involved in dopaminergic and serotoninergic systems are the candidate genes most frequently associated with IA [24]. Dopamine (DA) is an important monoamine that regulates various neural mechanisms, such as cognitive memory and emotional activity [25], and represents the final pathway of the reward system [26]. The availability of DA is regulated primarily by its transporter (DAT), which recaps dopamine at the level of nerve synapses [27]. The 3' untranslated region of the DAT1 gene contains a variable number of tandem repetitions (VNTRs) of 40 polymorphic base pairs, and the most frequent polymorphisms are 9 or 10 repetitions [28]. Neurobiological studies have shown a dysregulated dopamine transmission associated with IA [29,30], with a decreased level of expression of DAT in the striatum [31]. Another gene crucial to the dopaminergic system is the dopamine D4 receptor gene (DRD4), which is involved in a wide range of behavioral processes related to addiction, such as attention, motivation, and emotion [32]. This gene has a polymorphic 48-base pair VNTR in exon 3, and the most frequent polymorphisms are 4 (4R), 7 (7R), and 2 (2R) repeats [33]. Previous studies have underlined significant associations between the DRD4 4R genotype with increased attentiveness $[34,35]$ that, in turn, has been associated with a higher risk of IA [36]. Besides the role played by dopamine, it has been evidenced that serotonin may also be implicated in IA. Serotonin (5-HT) plays an important role in feelings of well-being, happiness, anxiety, stress susceptibility [37], and depression [38]. The serotonin-transporter-linked polymorphic region (5-HTTLPR) is crucial for the fine regulation of 5-HT [39]. This functional polymorphism results in 2 common alleles, the short (S) and the long (L) [40], and studies by Sun et al [36] and Lee et al [41] have reported significant association between IA and the $\mathrm{S} / \mathrm{S}$ genotype. Other studies involving allelic variants in monoamine oxidase A (MAO-A) activity have provided further support for the role played by serotonin and dopamine, given that this gene encodes an enzyme involved in the degradation of DA and 5-HT. The MAO-A gene has a 30-base pair repeat in the promoter region that affects transcriptional efficiency [42]. The high-activity variant (MAOA-H; 3.5 or 4 repeats) has shown significant association with impulsive personality traits [43] and tobacco and cannabis use [44], but other studies have reported conflicting results, with the low-activity variant (MAOA-L; 2, 3, or 5 repeats) associated with disordered gambling $[45,46]$. However, considering the complexity of IA, to date there is a dearth of studies that have considered possible interactions between genetic vulnerability and the risk provided by the social environment [47], although recent evidence has highlighted the genetic moderation on the impact of the environment on other disorders and behaviors related to addiction [48].

\section{The Role of Family Functioning}

In this field, the quality of family functioning has been suggested to be one of the main environmental risk factors for the onset and maintenance of IA among young adults [49,50]. In particular, many studies have shown that the lack of parental emotional support and connectedness, and the poor quality of the relationships with parents, have an important influence on the risk taking and addictive behaviors of their offspring [51-53], including IA [54-56]. Moreover, it has been posited that the poor quality of family functioning is associated with a wide range of psychopathological problems [57-59], which in turn may lead to a higher risk for the onset of IA [60].

\section{Individual Psychopathological Symptoms and IA}

International research has widely demonstrated the comorbidity between IA and other psychological difficulties, both in terms of psychopathological symptoms and personality disorder [61,62]. Although several studies have shown that this relationship may be reciprocal and bidirectional $[63,64]$, recent evidence has suggested that IA can be considered as the result of other psychopathological problems [20], resulting in a strategy to cope with psychological discomfort [2] and physical discomfort [65]. Depression and anxiety symptoms represent the 2 psychopathological areas most frequently associated with IA, both in clinical samples [66] and in the general population 
[67,68]. Moreover, significant associations with cluster C personality features have been evidenced [69], especially with traits of avoidant personality disorder [70].

\section{This Study}

From a biopsychosocial perspective [71], this exploratory study aims to evaluate the role played by biological, psychological, and social factors that may contribute to the etiology of IA in a sample of 104 young adults of the general population.

We hypothesized the presence of the following: (1) significant associations between IA and genetic polymorphisms in MAO-A (ie, MAOA-H genotype), 5-HTTPR (ie, S/S genotype), DRD4 (ie, 4R/4R genotype), and DAT1 (ie, 9-repeat genotype) genes, based on previous studies that have underlined the key role of these genes in the biochemistry of addiction disorder [45,72,73], including IA [36,41,74,75]; (2) significant differences in the quality of family functioning of young adults and psychological profiles (ie, depression and anxiety problems, avoidant personality problems) between youths with IA and the control group, as suggested by previous studies that have shown significant association between IA both with a poor quality of family functioning [49,50] and with psychopathological symptoms, especially in the areas of depression and anxiety symptoms [76,77] and traits of avoidant personality disorder [78]; (3) a mediation role played by the psychological profile of young adults on the relationship between family functioning and IA, based on studies that have shown a predictive effect of the quality of family functioning on the psychopathological profile of young adults $[57,58]$ that, in turn, has been suggested to be a significant predictor of IA [70]; and (4) the moderator role played by MAO-A, 5-HTTPR, DRD4, and DAT1 genotypes on the relationship between family functioning and IA, as suggested by recent evidence that has underlined that individual genotype play an important role in moderating individual sensitivity to environmental events $[79,80]$ and to the development of psychopathology related to addiction [48].

\section{Methods}

\section{Recruitment}

Over a period of 1 year, 150 young adults $(65 / 150,43.3 \%$ boys; $86 / 150,56.7 \%$ girls) aged between 19 and 23 years were recruited for this study, through the collaboration of universities in the center-south of Italy. All youths signed informed consent, in which the study was illustrated in detail. To examine the youths' genotype of the MAO-A, DAT1, 5-HTTPR, and DRD4 genes, we collected biological materials from human buccal swabs (the procedure is described below). In addition, the youths who agreed to participate in this study were administered self-report questionnaires (described below). This study was approved by the Ethical Committee of the Department of Dynamic and Clinical Psychology at Sapienza University of Rome, in accordance with the Declaration of Helsinki.

\section{Procedure for Biological Sampling}

Youths were assessed through buccal swabs (Isohelix Swab Pack) by a group of psychologists, specifically trained for the purposes of the study. Subjects were aware of not having to eat (including chewing gum, candy, etc), drink (except water), smoke, and brush their teeth for at least 1 hour before sampling. Epithelial cell samples were carefully collected through buccal swabs. The biological samplings were transported, slightly chilled by Normative ice $\left(+4^{\circ} \mathrm{C}\right)$, to the laboratories of the co-author, EP, for further processing. After buccal swabs were gathered, young adults filled self-report questionnaires (described below).

\section{Methods}

\section{Assessment of Internet Use or Abuse Among Young Adults}

The IA test (IAT) [81] is a 20-item, 5-point Likert scale that measures the severity of self-reported compulsive use of the internet. Total IA scores were calculated, with possible scores ranging from 20 to 100 . The scale showed very good internal consistency, with a Cronbach $\alpha$ value of .82 in this study. According to Italian validation [82], total IAT from 0 to 39 represents average users with complete control of their internet use, scores from 40 to 69 represent excessive internet use, and scores from 70 to 100 represent significant problems because of internet use.

\section{Assessment of Psychological Profiles of Young Adults}

The Adult Self-Report (ASR) [83] is a self-report used to elicit information regarding psychological functioning. Items are assessed on a 3 -point Likert scale $(0=$ not true, $1=$ sometimes true, and $2=$ very often true). The aim of this study is to explore the role played by depression, anxiety, and avoidant personality problems of young adults on IA. Consequently, we used the scores of the following DSM-oriented scales: depressive problems, anxiety problems, and avoidant personality problems. Research has demonstrated good reliability and validity for the scales of the ASR [83]. In this study, the ASR showed good internal coherence (Cronbach $\alpha$ alpha=.73-.84).

\section{Assessment of Family Functioning of Young Adults}

The Family Assessment Device (FAD) $[84,85]$ is a self-report questionnaire that was developed to measure perceptions of family functioning. It is composed of 60 items evaluated on a 4 -point scale ( $1=$ strongly agree and $4=$ strongly disagree), and measures the 6 dimensions of the McMaster Model of Family Functioning: (1) problem solving, which refers to the family's ability to solve problems; (2) communication, which refers to whether communication in the family is clear and direct or vague and indirect; (3) roles, which addresses the issue of how roles and responsibilities are allocated among family members; (4) affective responsiveness (AR), which refers to the ability of the family members to respond to a range of situations with appropriate quality and amount of emotion; (5) affective involvement refers to how family members experience interest in and involvement with each other; and (6) behavioral control, which assesses whether the family has norms or standards governing individual behavior and responses to emergency situations. In all dimensions, higher scores represent less satisfaction with family functioning. The validity, reliability, and internal consistency of individual scales have been thoroughly investigated [86]. The internal consistency of the 6 subscales in this study was also adequate (Cronbach $\alpha=.77-.87$ ). 


\section{DNA Isolation and Genotyping}

Buccal cell DNA isolations were performed using the Buccal Prep Plus DNA isolation kit (Isohelix) according to the manufacturer's instructions. The yield of DNA is usually between 3 and $10 \mu \mathrm{g}$. Allelic variants of the MAO-A, DAT1, 5-HTTPR, and DRD4 genes were identified. Genotypes were grouped into dominant, recessive, and additive genetic models, with the exception of MAO-A among males. The MAO-A gene is located on the $\mathrm{X}$ chromosome, so males have 1 allelic variant. Consequently, for males, the MAO-A gene was grouped based on the presence of the MAOA-H activity allele (ie, 4R) and the MAOA-L activity alleles (ie, 2R, 3R, and 5R). For females, the MAO-A gene was grouped based on the presence of 2 copies of the MAO-H allele, at least one copy of the MAO-H allele or 2 copies of the MAO-L allele. For the DRD4 gene, previous studies [87,88] have emphasized that the $4 \mathrm{R}$ allele is the ancestral allele and has distinct functionality with regard to other alleles. Consequently, subjects were grouped based on the presence of 2 copies of the $4 \mathrm{R}$ allele, at least one copy of the $4 \mathrm{R}$ allele, and without the $4 \mathrm{R}$ allele. With regard to the 5-HTTPR genotype, we considered the presence of 2 copies of the $\mathrm{S}$ allele (ie, $\mathrm{S} / \mathrm{S}$ ), at least one copy of the $\mathrm{S}$ allele (ie, $\mathrm{S} / \mathrm{L}$ ), and 2 copies of the $\mathrm{L}$ allele (ie, L/L). Finally, for the DAT1 gene, groups were formed based on the presence of 2 copies of the 10R allele (ie, 10/10), at least one copy of the 10R allele (ie, 9/10), and 2 copies of the $9 \mathrm{R}$ allele.

\section{Statistical Analysis}

Preliminary analyses were performed using descriptive statistics (frequencies, percentages, and mean scores). Deviations from Hardy-Weinberg equilibrium were tested using the chi-square test, which allows the comparison of the expected frequency of the specific polymorphism with the observed one [89]. The Hardy-Weinberg equilibrium of the MAO-A among males was tested using the method proposed by Graffelman and Weir [90] for biallelic variants on the $\mathrm{X}$ chromosome, which considers both males and females. To verify the possible association between IA (IA group vs control group) and genetic polymorphisms in MAO-A, 5-HTTPR, DRD4, and DAT1 genes, considering the role played by gender, sex-stratified logistic-regression models were conducted. For each marker, we examined the dominant, recessive, and additive genetic models. An allelic model was used to examine the possible effect of MAO-A among males. Odds ratios and 95\% CIs are presented. To verify the possible differences between the 2 groups on all the FAD subscales and on the ASR scores of the DSM-oriented subscales of depressive problems, anxiety problems, and avoidant personality problems, two tailed $t$ test for independent samples was carried out. Based on preliminary analyses, a total sample bootstrap mediation analysis for simple and multiple mediation was then conducted to explore the possible mediation effects of the emotional-behavioral functioning of young adults on the relationship between the perception of young adults of their family functioning and their scores on IAT. The scores of the independent variable and of the mediators were standardized before performing the mediation analyses. Indirect (ie, mediating) effects were evaluated with $95 \%$ bias-corrected CI based on 5000 bootstrap samples. Finally, we tested whether the relationship between FAD and IAT could be moderated by genetic polymorphisms in the MAO-A, 5-HTTPR, DRD4, and DAT1 genes in young adults. For moderation analyses, only dominant genetic models were used. We standardized the score of the independent variable before performing the moderation analyses. All analyses were performed using IBM SPSS software 25.0. Mediation and moderation analyses were performed using PROCESS for SPSS [91].

\section{Results}

\section{Sample Characteristics}

In this study, from the total sample of 150 youths, $16.6 \%$ $(25 / 150)$ of youths who did not complete the assessment procedure and $12.6 \%(19 / 150)$ of youths for whom it was not possible to collect biological samples were excluded. The final sample consisted of 104 young adults (mean age 21.87, SD 2.29 years; 50/104, 48.1\% males). All youths recruited were university students. Most of the youths recruited for the study lived in families $(101 / 104,97.1 \%)$. Of the parents, $88.5 \%$ $(92 / 104)$ were married or cohabiting, whereas $11.5 \%(12 / 104)$ were separated. On the basis of the IAT cut-off for Italian validation $(\geq 40)$ [82], for aims 1 and 2 of this study, the total sample was divided into 2 subgroups: (1) IA group: comprising youths who reported excessive internet use (35/104, 33.6\%) and (2) control group: comprising youths in complete control of their internet use $(69 / 104,66.4 \%)$.

The allele and genotype frequencies of the DRD4, MAO-A, 5-HTTPR, and DAT1 genes among males (Table 1) and females (Table 2) are reported in this study.

Genotypes were distributed according to the Hardy-Weinberg equilibrium among male (DRD4: $\chi^{2}{ }_{1}=2.1, P=.14$; 5HTTPR: $\chi_{1}^{2}=.07, P=.78$; DAT1: $\chi_{1}^{2}=1.2, P=.26$ ) and female (DRD4: $\chi_{1}^{2}=.04, P=.83$; 5HTTPR: $\chi_{1}{ }_{1}=.1, P=.65$; DAT $\left.1: \chi_{1}^{2}=.4, P=.48\right)$ youths, with the exception of MAO-A (overall: $\chi^{2}{ }_{3}=7.8, P=.05$; females: $\left.\chi_{3}^{2}=7.1, P=.007\right)$. 
Table 1. Allele and genotype frequencies of the dopamine D4 receptor, monoamine oxidase A, serotonin-transporter, and dopamine active transporter 1 genes among males.

\begin{tabular}{|c|c|c|c|c|}
\hline Genes & $\mathrm{DRD}^{\mathrm{a}}, \mathrm{n}(\%)$ & MAO-A ${ }^{b}, \mathrm{n}(\%)$ & 5-HTTLPR ${ }^{\mathrm{c}}, \mathrm{n}(\%)$ & $\mathrm{DAT}^{\mathrm{d}}, \mathrm{n}(\%)$ \\
\hline \multicolumn{5}{|l|}{ Allele } \\
\hline 2 & $5(5)$ & $0(0)$ & $\mathrm{N} / \mathrm{A}^{\mathrm{e}}$ & N/A \\
\hline 3 & $3(3)$ & $16(32)$ & N/A & N/A \\
\hline 4 & $72(72)$ & $33(66)$ & N/A & N/A \\
\hline 5 & $3(3)$ & $1(2)$ & N/A & N/A \\
\hline 6 & $1(1)$ & N/A & N/A & N/A \\
\hline 7 & $5(5)$ & N/A & N/A & N/A \\
\hline 8 & $4(4)$ & N/A & N/A & N/A \\
\hline $\mathrm{L}$ & N/A & N/A & $48(48)$ & N/A \\
\hline$S$ & N/A & N/A & $52(52)$ & N/A \\
\hline 9 & N/A & N/A & N/A & $32(32)$ \\
\hline 10 & N/A & N/A & N/A & $62(62)$ \\
\hline \multicolumn{5}{|l|}{ Genotype } \\
\hline $2 \mathrm{R} / 2 \mathrm{R}$ & $1(2)$ & N/A & N/A & N/A \\
\hline $2 \mathrm{R} / 4 \mathrm{R}$ & $8(16)$ & N/A & N/A & N/A \\
\hline $2 \mathrm{R} / 5 \mathrm{R}$ & $1(2)$ & N/A & N/A & N/A \\
\hline $2 \mathrm{R} / 7 \mathrm{R}$ & $1(2)$ & N/A & N/A & N/A \\
\hline $3 R / 3 R$ & $0(0)$ & N/A & N/A & N/A \\
\hline $3 \mathrm{R} / 4 \mathrm{R}$ & $2(4)$ & N/A & N/A & N/A \\
\hline $3 \mathrm{R} / 7 \mathrm{R}$ & $0(0)$ & N/A & N/A & N/A \\
\hline $3 \mathrm{R} / 8 \mathrm{R}$ & $1(2)$ & N/A & N/A & N/A \\
\hline $4 \mathrm{R} / 4 \mathrm{R}$ & $28(56)$ & N/A & N/A & N/A \\
\hline $4 \mathrm{R} / 5 \mathrm{R}$ & $2(4)$ & N/A & N/A & N/A \\
\hline $4 \mathrm{R} / 7 \mathrm{R}$ & $2(2)$ & N/A & N/A & N/A \\
\hline $4 \mathrm{R} / 8 \mathrm{R}$ & $3(6)$ & N/A & N/A & N/A \\
\hline $5 \mathrm{R} / 7 \mathrm{R}$ & $0(0)$ & N/A & N/A & N/A \\
\hline $6 \mathrm{R} / 7 \mathrm{R}$ & $1(2)$ & N/A & N/A & N/A \\
\hline 7R/7R & $1(2)$ & N/A & N/A & N/A \\
\hline $\mathrm{L} / \mathrm{L}$ & N/A & N/A & $12(24)$ & N/A \\
\hline $\mathrm{L} / \mathrm{S}$ & N/A & N/A & $24(48)$ & N/A \\
\hline $\mathrm{S} / \mathrm{S}$ & N/A & N/A & $14(28)$ & N/A \\
\hline $9 / 9$ & N/A & N/A & N/A & $5(12)$ \\
\hline $9 / 10$ & N/A & N/A & N/A & $27(42)$ \\
\hline $10 / 10$ & N/A & N/A & N/A & $18(36)$ \\
\hline
\end{tabular}

${ }^{\mathrm{a}} \mathrm{DRD} 4$ : dopamine D4 receptor gene.

${ }^{\mathrm{b}} \mathrm{MAO}-\mathrm{A}$ : monoamine oxidase A.

${ }^{{ }^{5} 5-H T T L P R: ~ s e r o t o n i n-t r a n s p o r t e r-l i n k e d ~ p o l y m o r p h i c ~ r e g i o n . ~}$

${ }^{\mathrm{d} D A T 1: \text { dopamine transporter } 1 .}$

${ }^{\mathrm{e}} \mathrm{N} / \mathrm{A}$ : not applicable. 
Table 2. Allele and genotype frequencies of the dopamine D4 receptor, monoamine oxidase A, serotonin-transporter, and dopamine active transporter 1 genes among females.

\begin{tabular}{|c|c|c|c|c|}
\hline Genes & $\mathrm{DRD}^{\mathrm{a}}, \mathrm{n}(\%)$ & 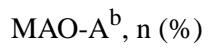 & 5-HTTLPR ${ }^{\mathrm{c}}, \mathrm{n}(\%)$ & $\mathrm{DAT}^{\mathrm{d}}, \mathrm{n}(\%)$ \\
\hline \multicolumn{5}{|l|}{ Allele } \\
\hline 2 & $7(6.5)$ & $2(1.8)$ & $\mathrm{N} / \mathrm{A}^{\mathrm{e}}$ & N/A \\
\hline 3 & $11(10.2)$ & $33(30.6)$ & N/A & N/A \\
\hline 4 & $84(77.7)$ & $68(63)$ & N/A & N/A \\
\hline 5 & $3(2.7)$ & $5(4.6)$ & N/A & N/A \\
\hline 6 & $0(0)$ & N/A & N/A & N/A \\
\hline 7 & $3(2.7)$ & N/A & N/A & N/A \\
\hline 8 & $0(0)$ & N/A & N/A & N/A \\
\hline $\mathrm{L}$ & N/A & N/A & $62(57.4)$ & N/A \\
\hline S & N/A & N/A & $46(42.6)$ & N/A \\
\hline 9 & N/A & N/A & N/A & $42(38.9)$ \\
\hline 10 & N/A & N/A & N/A & $66(61.1)$ \\
\hline \multicolumn{5}{|l|}{ Genotype } \\
\hline $2 \mathrm{R} / 2 \mathrm{R}$ & $1(1.9)$ & $1(1.9)$ & N/A & N/A \\
\hline $2 \mathrm{R} / 4 \mathrm{R}$ & $5(9.3)$ & N/A & N/A & N/A \\
\hline $2 \mathrm{R} / 5 \mathrm{R}$ & $0(0)$ & N/A & N/A & N/A \\
\hline $2 \mathrm{R} / 7 \mathrm{R}$ & $0(0)$ & N/A & N/A & N/A \\
\hline $3 \mathrm{R} / 3 \mathrm{R}$ & $3(5.6)$ & $9(16.7)$ & N/A & N/A \\
\hline $3 \mathrm{R} / 4 \mathrm{R}$ & $3(5.6)$ & $15(27.8)$ & N/A & N/A \\
\hline $3 \mathrm{R} / 7 \mathrm{R}$ & $2(3.7)$ & N/A & N/A & N/A \\
\hline $3 \mathrm{R} / 8 \mathrm{R}$ & $0(0)$ & N/A & N/A & N/A \\
\hline $4 R / 4 R$ & 37 (68.5) & $26(48.1)$ & N/A & N/A \\
\hline $4 \mathrm{R} / 5 \mathrm{R}$ & $2(3.7)$ & $1(1.9)$ & N/A & N/A \\
\hline $4 \mathrm{R} / 7 \mathrm{R}$ & $0(0)$ & N/A & N/A & N/A \\
\hline $4 \mathrm{R} / 8 \mathrm{R}$ & $0(0)$ & N/A & N/A & N/A \\
\hline $5 \mathrm{R} / 5 \mathrm{R}$ & N/A & $2(3.7)$ & N/A & N/A \\
\hline $5 \mathrm{R} / 7 \mathrm{R}$ & $1(1.9)$ & N/A & N/A & N/A \\
\hline $6 \mathrm{R} / 7 \mathrm{R}$ & $0(0)$ & N/A & N/A & N/A \\
\hline 7R/7R & $0(0)$ & N/A & N/A & N/A \\
\hline $\mathrm{L} / \mathrm{L}$ & N/A & N/A & 17 (31.5) & N/A \\
\hline $\mathrm{L} / \mathrm{S}$ & N/A & N/A & $28(51.9)$ & N/A \\
\hline $\mathrm{S} / \mathrm{S}$ & N/A & N/A & $9(16.7)$ & N/A \\
\hline $9 / 9$ & N/A & N/A & N/A & $9(16.6)$ \\
\hline $9 / 10$ & N/A & N/A & N/A & $23(42.6)$ \\
\hline $10 / 10$ & N/A & N/A & N/A & $22(40.8)$ \\
\hline
\end{tabular}

${ }^{\mathrm{a} D R D} 4$ : dopamine $\mathrm{D} 4$ receptor gene.

${ }^{b}$ MAO-A: monoamine oxidase A.

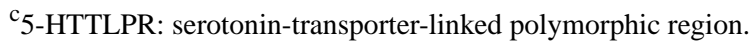

${ }^{\mathrm{d} D A T 1:}$ dopamine transporter 1.

${ }^{\mathrm{e}} \mathrm{N} / \mathrm{A}$ : not applicable. 
Association Between IA and Genetic Polymorphisms

To verify the possible association between IA and genetic polymorphisms in MAO-A, 5-HTTPR, DRD4, and DAT1 genes among male and female youths, sex-stratified logistic-regression models were conducted. If the CI crosses 1, the difference between genotype groups can be considered not significant. As shown in Tables 3 and 4, no significant associations were found between IA and any considered genotypes, both among males and females (all CIs crossed 1).

Table 3. Association between internet addiction and genetic polymorphisms in serotonin-transporter, dopamine active transporter 1, dopamine D4 receptor, and monoamine oxidase $\mathrm{A}$ among male youths.

\begin{tabular}{|c|c|c|c|}
\hline Gene and genotype & Wald $\chi^{2}(d f)$ & $P$ value & $\mathrm{OR}^{\mathrm{a}}(95 \% \mathrm{CI})$ \\
\hline \multicolumn{4}{|l|}{ 5-HTTPR ${ }^{b}$} \\
\hline $\mathrm{S} / \mathrm{S}$ vs $\mathrm{S} / \mathrm{L}+\mathrm{L} / \mathrm{L}^{\mathrm{c}}$ & $0.5(1)$ & .47 & $1.57(0.45-5.45)$ \\
\hline $\mathrm{L} / \mathrm{L}$ vs $\mathrm{S} / \mathrm{S}+\mathrm{S} / \mathrm{L}^{\mathrm{d}}$ & $0.001(1)$ & .97 & $0.98(0.26-3.66)$ \\
\hline $\mathrm{S} / \mathrm{S}^{\mathrm{e}}$ & $0.5(2)$ & .75 & $N / A^{f}$ \\
\hline $\mathrm{S} / \mathrm{L}$ & $0.05(1)$ & .67 & $1.4(0.29-6.62)$ \\
\hline $\mathrm{L} / \mathrm{L}$ & $0.3(1)$ & .80 & $0.84(0.20-3.45)$ \\
\hline \multicolumn{4}{|l|}{ DAT1 $^{g}$} \\
\hline $10 / 10$ vs $9 / 10+9 / 9^{c}$ & $0.1(1)$ & .73 & $0.81(0.25-2.65)$ \\
\hline $9 / 9$ vs $9 / 10+10 / 10^{\mathrm{d}}$ & $0.7(1)$ & .39 & $2.25(0.34-14.83)$ \\
\hline $10 / 10^{\mathrm{e}}$ & $0.7(2)$ & .69 & N/A \\
\hline $9 / 10$ & $0.6(1)$ & .40 & $0.42(0.05-3.21)$ \\
\hline $9 / 9$ & $0.6(1)$ & .43 & $0.45(0.06-3.21)$ \\
\hline \multicolumn{4}{|l|}{ DRD4 ${ }^{h}$} \\
\hline $4 / 4$ vs non $4 / 4^{c}$ & $0.5(1)$ & .47 & $0.65(0.21-2.06)$ \\
\hline \multicolumn{4}{|l|}{ MAO-A ${ }^{\mathbf{i}}$} \\
\hline$H$ vs $L^{j}$ & $0.007(1)$ & .93 & $0.95(0.29-3.11)$ \\
\hline L vs $\mathrm{H}$ & $0.007(1)$ & .93 & $1.05(0.32-3.45)$ \\
\hline
\end{tabular}

${ }^{\mathrm{a} O R}$ : odds ratio.

${ }^{b}$ 5-HTTLPR: serotonin-transporter-linked polymorphic region.

${ }^{\mathrm{c}}$ Dominant.

${ }^{\mathrm{d}}$ Recessive.

eAdditive.

${ }^{\mathrm{f}} \mathrm{N} / \mathrm{A}$ : not applicable.

gDAT1: dopamine transporter 1.

${ }^{\mathrm{h}}$ DRD4: dopamine D4 receptor gene.

${ }^{\mathrm{i}} \mathrm{MAO}-\mathrm{A}$ : monoamine oxidase A.

${ }^{\mathrm{j}}$ Allelic. 
Table 4. Association between internet addiction and genetic polymorphisms in serotonin-transporter, dopamine active transporter 1, dopamine D4 receptor, and monoamine oxidase A among female youths.

\begin{tabular}{|c|c|c|c|}
\hline Gene and genotype & Wald $\chi^{2}(d f)$ & $P$ value & $\mathrm{OR}^{\mathrm{a}}(95 \% \mathrm{CI})$ \\
\hline \multicolumn{4}{|l|}{ 5-HTTPR ${ }^{b}$} \\
\hline $\mathrm{S} / \mathrm{S}$ vs $\mathrm{S} / \mathrm{L}+\mathrm{L} / \mathrm{L}^{\mathrm{c}}$ & $0.07(1)$ & .78 & $0.78(0.14-4.32)$ \\
\hline $\mathrm{L} / \mathrm{L}$ vs $\mathrm{S} / \mathrm{S}+\mathrm{S} / \mathrm{L}^{\mathrm{d}}$ & $0.1(1)$ & .69 & $1.29(0.35-4.68)$ \\
\hline $\mathrm{S} / \mathrm{S}^{\mathrm{e}}$ & $0.1(2)$ & .91 & $N / A^{f}$ \\
\hline $\mathrm{S} / \mathrm{L}$ & $0.1(1)$ & .69 & $0.68(0.10-4.52)$ \\
\hline $\mathrm{L} / \mathrm{L}$ & $0.1(1)$ & .74 & $0.80(0.20-3.08)$ \\
\hline \multicolumn{4}{|l|}{ DAT1 ${ }^{g}$} \\
\hline $10 / 10$ vs $9 / 10+9 / 9^{c}$ & $1.1(1)$ & .28 & $0.48(0.13-1.82)$ \\
\hline $9 / 9$ vs $9 / 10+10 / 10^{d}$ & $0.0(1)$ & .78 & $0.78(0.14-4.32)$ \\
\hline $10 / 10^{\mathrm{e}}$ & $1.6(2)$ & .43 & N/A \\
\hline $9 / 10$ & $0.06(1)$ & .79 & $0.77(0.11-5.24)$ \\
\hline $9 / 9$ & $0.4(1)$ & .49 & $1.86(0.31-11.18)$ \\
\hline \multicolumn{4}{|l|}{ DRD4 ${ }^{h}$} \\
\hline $4 / 4$ vs non $4 / 4^{c}$ & $0.4(1)$ & .48 & $1.55(0.44-5.42)$ \\
\hline \multicolumn{4}{|l|}{ MAO-A ${ }^{\mathrm{i}}$} \\
\hline $\mathrm{H} / \mathrm{H}$ vs $\mathrm{H} / \mathrm{L}+\mathrm{L} / \mathrm{L}^{\mathrm{c}}$ & $0.2(1)$ & .64 & $0.75(0.22-2.55)$ \\
\hline $\mathrm{L} / \mathrm{L}$ vs $\mathrm{H} / \mathrm{H}+\mathrm{H} / \mathrm{L}^{\mathrm{d}}$ & $0.6(1)$ & .41 & $0.50(0.09-2.62)$ \\
\hline $\mathrm{H} / \mathrm{H}^{\mathrm{e}}$ & $1.7(2)$ & .42 & N/A \\
\hline $\mathrm{H} / \mathrm{L}$ & $0.2(1)$ & .65 & $0.66(0.11-3.91)$ \\
\hline $\mathrm{L} / \mathrm{L}$ & $0.9(1)$ & .31 & $2(0.51-7.81)$ \\
\hline
\end{tabular}

${ }^{\mathrm{a}} \mathrm{OR}$ : odds ratio.

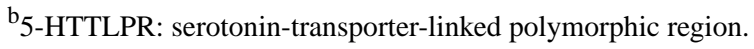

${ }^{\mathrm{c}}$ Dominant.

${ }^{\mathrm{d}}$ Recessive.

${ }^{\mathrm{e}}$ Additive.

${ }^{\mathrm{f}} \mathrm{N} / \mathrm{A}$ : not applicable.

${ }^{\mathrm{g}}$ DAT1: dopamine transporter 1.

${ }^{\mathrm{h}}$ DRD4: dopamine D4 receptor gene.

${ }^{\mathrm{i}} \mathrm{MAO}-\mathrm{A}$ : monoamine oxidase A.

\section{IA, Family Functioning, and Psychopathological Symptoms of Young Adults}

To verify possible differences between the 2 groups in family functioning and the psychological profile of young adults (ie, depression, anxiety, and avoidant personality problems), an independent samples $t$ test was conducted. Results showed significant differences between youths with IA and their peers in the control group in the scores of the FAD AR subscale scores and ASR scores in all psychopathological areas (Table 5). 
Table 5. $\mathrm{t}$ test for family functioning and psychological profile for the 2 groups.

\begin{tabular}{|c|c|c|c|c|}
\hline Groups or study variables & $\mathrm{IAG}^{\mathrm{a}}(\mathrm{n}=35)$, mean $(\mathrm{SD})$ & $\mathrm{CG}^{\mathrm{b}}(\mathrm{n}=69)$, mean $(\mathrm{SD})$ & $t$ test $(d f)$ & $P$ value \\
\hline \multicolumn{5}{|l|}{ FAD $^{\mathbf{c}}$} \\
\hline $\mathrm{PS}^{\mathrm{d}}$ & $13.60(2.95)$ & $12.98(3.36)$ & $0.91(102)$ & .36 \\
\hline $\mathrm{CM}^{\mathrm{e}}$ & $20.31(2.97)$ & $19.36(2.69)$ & $1.64(102)$ & .10 \\
\hline$R L^{f}$ & $25.97(2.13)$ & $26,27(2.02)$ & $-0.71(102)$ & .47 \\
\hline $\mathrm{AR}^{\mathrm{g}}$ & $15.25(2.47)$ & $13.95(2.62)$ & $2.43(102)$ & .01 \\
\hline $\mathrm{AI}^{\mathrm{h}}$ & $18.25(2.01)$ & $18.59(1.44)$ & $-0.97(102)$ & .33 \\
\hline $\mathrm{BC}^{\mathrm{i}}$ & $21.51(2.09)$ & $20.73(2.48)$ & $1.58(102)$ & .11 \\
\hline \multicolumn{5}{|l|}{$\mathbf{A S R}^{\mathbf{j}}$} \\
\hline Depression & $7.80(5.08)$ & $5.66(4.16)$ & $2.28(102)$ & .02 \\
\hline Anxiety & $7.25(2.59)$ & $5.94(2.24)$ & $2.68(102)$ & .009 \\
\hline Avoidant personality & $4.94(2.85)$ & $3.27(1.90)$ & $3.11(102)$ & .003 \\
\hline
\end{tabular}

${ }^{\mathrm{a}} \mathrm{IAG}$ : internet addiction group.

${ }^{\mathrm{b}} \mathrm{CG}$ : control group.

${ }^{\mathrm{c}}$ FAD: Family Assessment Device.

${ }^{\mathrm{d}}$ PS: problem solving.

${ }^{\mathrm{e}} \mathrm{CM}$ : communication.

${ }^{\mathrm{f}} \mathrm{RL}$ : roles.

${ }^{\mathrm{g}} \mathrm{AR}$ : affective responsiveness.

${ }^{\mathrm{h}} \mathrm{AI}$ : affective involvement.

${ }^{\mathrm{i}} \mathrm{BC}$ : behavioral control.

${ }^{\mathrm{j}}$ ASR: Adult Self-Report.

\section{The Mediation Role Played by the Psychological Profile on the Relationship Between Family Functioning and IA}

On the basis of the previous analyses, we verified the possible simple and multiple mediation effects of depressive and anxiety symptoms and avoidant personality traits on the relationship between FAD AR and IA. Mediation analyses were performed using the SPSS Macro PROCESS [91], which provides coefficient estimates for the total, direct, and indirect effects of variables using ordinary least squares regression. In particular,
Model 4 [91] was used, which allows us to estimate the indirect effects within a $95 \%$ CI. The indirect effect can be considered statistically significant if the CI does not include zero. In our first simple mediation model, we tested whether youth's depressive symptoms mediated the effect of FAD AR on youth's IA. Results showed that FAD AR was significantly associated with youth depressive symptoms $(B=0.29 ; P=.002)$, which, in turn, was positively related to the youth's scores on IAT $(B=3.33 ; P<.001)$. Moreover, considering the relationship between FAD AR and IAT, both direct $(B=1.90 ; P=.04)$ and total effect $(B=2.90 ; P=.002)$ were significant (Figure 1). 
Figure 1. Simple mediation model for depressive problems mediating the association between affective responsiveness and internet addiction. (a) Direct effect of affective responsiveness on depression; (b) direct effect of depression on internet addiction test (IAT) score; (c') direct effect of affective responsiveness on IAT score; (c) total effect $\left(\mathrm{a}^{*} \mathrm{~b}+\mathrm{c}^{\prime}\right)$ of affective responsiveness on IAT score. ASR: Adult Self-Report; FAD: Family Assessment Device; IAT: internet addiction test.

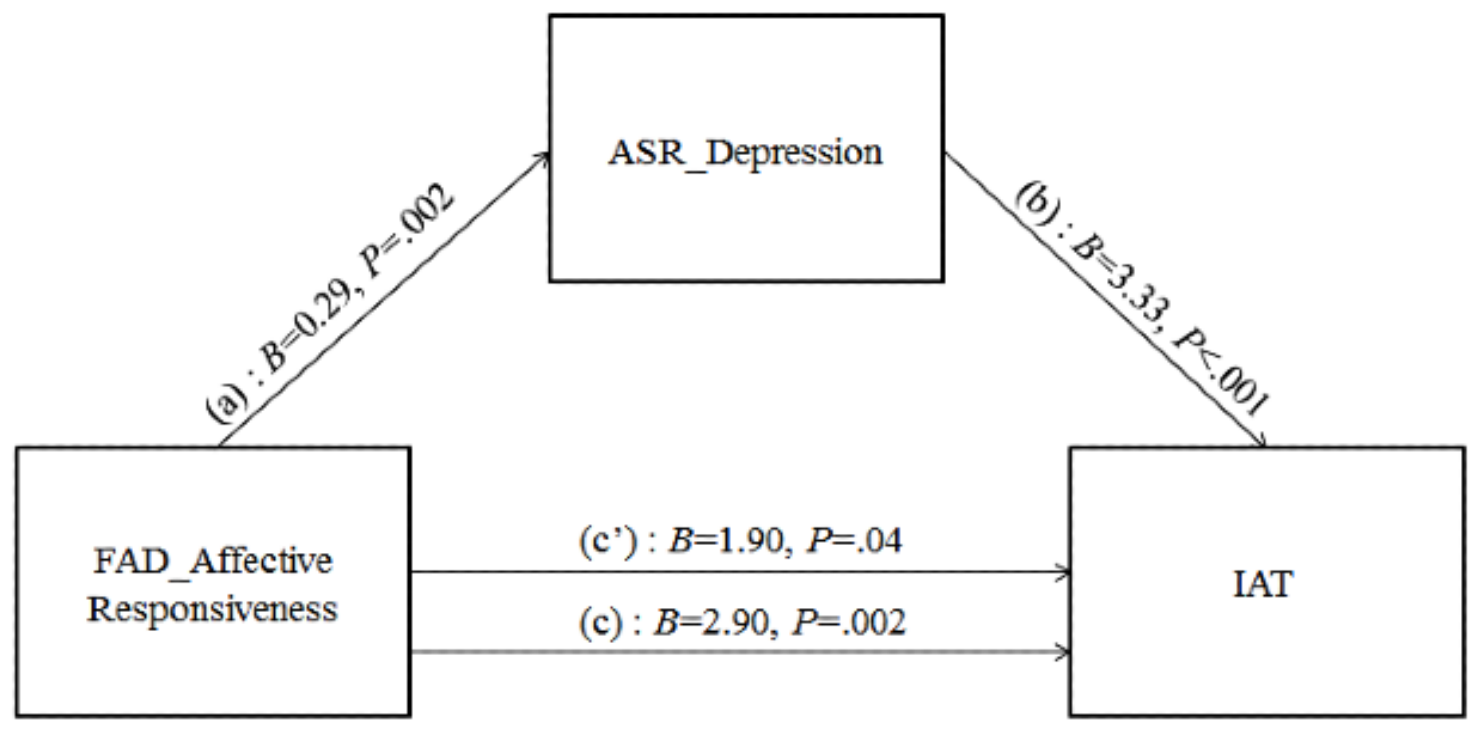

With regard to the indirect effect, the bootstrap CI showed that the indirect path via depression was statistically significant (Table 6).

We then tested whether the anxiety symptoms of young adults mediated the relationships between the FAD AR and IA. The results showed that the indirect effect of FAD AR through anxiety symptoms of young adults was not significant (Table 6). However, both direct $(B=2.50 ; P=.008)$ and total effect $(B=2.90 ; P=.002)$ of FAD AR on IAT were significant. Moreover, anxiety problems of young adults were significantly associated with their scores on IAT $(B=2.61 ; P=.005)$, but the relationship between FAD AR and depression in young adults was not significant ( $P=.12$; Figure 2$)$.

In relation to the possible mediation role played by avoidant personality traits of young adults on the relationship between FAD AR and IA, results showed that FAD AR was significantly related to their avoidant personality traits $(B=0.32 ; P<.001)$ that, in turn, was significantly associated with the IA of young adults $(B=3.38 ; P<.001$; Figure 3$)$. 
Table 6. Simple mediator models showing total, direct, and indirect effects of affective responsiveness on internet addiction.

\begin{tabular}{|c|c|c|}
\hline \multirow[t]{2}{*}{ Psychopathological areas and paths } & \multicolumn{2}{|l|}{ Path effect } \\
\hline & $\mathrm{B}(\mathrm{SE})$ & $95 \% \mathrm{CI}$ \\
\hline \multicolumn{3}{|l|}{ Depression } \\
\hline \multicolumn{3}{|l|}{ Direct path } \\
\hline $\operatorname{AfRes}^{\mathrm{a}} \rightarrow \mathrm{DEP}^{\mathrm{b}}$ & $0.29(0.09)$ & 0.10 to 0.48 \\
\hline $\mathrm{DEP} \rightarrow \mathrm{IAT}^{\mathrm{c}}$ & $3.33(0.94)$ & 1.46 to 5.20 \\
\hline AfRes $\rightarrow$ IAT & $1.90(0.94)$ & 0.04 to 3.77 \\
\hline \multicolumn{3}{|l|}{ Indirect path } \\
\hline AfRes $\rightarrow$ DEP $\rightarrow$ IAT & $0.99\left(0.44^{\mathrm{d}}\right)$ & 0.22 to $1.97^{\mathrm{e}}$ \\
\hline \multicolumn{3}{|l|}{ Total $^{\mathbf{f}}$} \\
\hline AfRes $\rightarrow$ IAT & $2.90(0.94)$ & 1.01 to 4.78 \\
\hline \multicolumn{3}{|l|}{ Anxiety } \\
\hline \multicolumn{3}{|l|}{ Direct path } \\
\hline AfRes $\rightarrow$ ANX $^{\mathrm{g}}$ & $0.15(0.09)$ & -0.04 to 0.34 \\
\hline ANX $\rightarrow$ IAT & $2.61(0.92)$ & 0.77 to 4.46 \\
\hline AfRes $\rightarrow$ IAT & $2.50(0.92)$ & 0.66 to 4.34 \\
\hline \multicolumn{3}{|l|}{ Indirect path } \\
\hline AfRes $\rightarrow$ ANX $\rightarrow$ IAT & $0.39\left(0.35^{\mathrm{d}}\right)$ & -0.11 to $1.24^{\mathrm{e}}$ \\
\hline \multicolumn{3}{|l|}{ Total $^{h}$} \\
\hline AfRes $\rightarrow$ IAT & $2.90(0.94)$ & 1.01 to 4.78 \\
\hline \multicolumn{3}{|l|}{ Avoidant personality } \\
\hline \multicolumn{3}{|l|}{ Direct path } \\
\hline AfRes $\rightarrow \mathrm{AvP}^{\mathrm{i}}$ & $0.32(0.09)$ & 0.13 to 0.50 \\
\hline $\mathrm{AvP} \rightarrow \mathrm{IAT}$ & $3.38(0.94)$ & 1.49 to 5.26 \\
\hline AfRes $\rightarrow$ IAT & $1.80(0.94)$ & -0.07 to 3.69 \\
\hline \multicolumn{3}{|l|}{ Indirect path } \\
\hline AfRes $\rightarrow$ AvP $\rightarrow$ IAT & $1.09\left(0.45^{\mathrm{d}}\right)$ & 0.32 to $2.05^{\mathrm{e}}$ \\
\hline \multicolumn{3}{|l|}{ Total $^{j}$} \\
\hline AfRes $\rightarrow$ IAT & $2.90(0.94)$ & 1.01 to 4.78 \\
\hline
\end{tabular}

afRes: affective responsiveness.

bDEP: Depression.

${ }^{\mathrm{c}}$ IAT: internet addiction test.

${ }^{\mathrm{d}}$ Bootstrapped SE values.

${ }^{\mathrm{e}}$ Bootstrap CI values.

${ }^{\mathrm{f}}$ Proportion mediated by indirect effect is $0.34 \%$.

${ }^{g}$ ANX: Anxiety.

${ }^{\mathrm{h}}$ Proportion mediated by indirect effect is $0.13 \%$.

${ }^{\mathrm{i}}$ AvP: avoidant personality.

${ }^{\mathrm{j}}$ Proportion mediated by indirect effect is $0.37 \%$. 
Figure 2. Simple mediation model for anxiety problems mediating the association between affective responsiveness and internet addiction. (a) Direct effect of affective responsiveness on anxiety problems; (b) direct effect of anxiety problems on IAT score; (c') direct effect of affective responsiveness on IAT score; (c) total effect $\left(\mathrm{a}^{*} \mathrm{~b}+\mathrm{c}^{\prime}\right)$ of affective responsiveness on IAT score. ASR: Adult Self-Report; FAD: Family Assessment Device; IAT: internet addiction test.

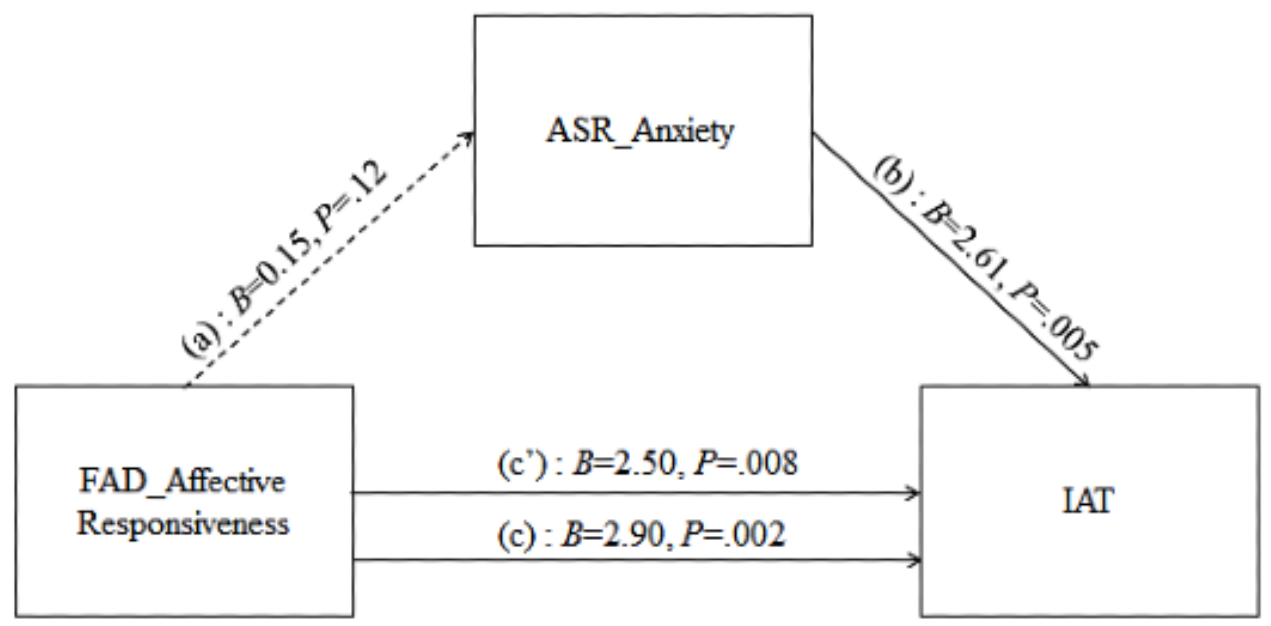

Figure 3. Simple mediation model for avoidant personality problems mediating the association between affective responsiveness and internet addiction. (a) Direct effect of affective responsiveness on avoidant personality traits; (b) direct effect of avoidant personality traits on IAT score; (c') direct effect of affective responsiveness on IAT score; (c) total effect ( $\left.a^{*} b+c^{\prime}\right)$ of affective responsiveness on IAT score. ASR: Adult Self-Report; FAD: Family Assessment Device; IAT: internet addiction test.

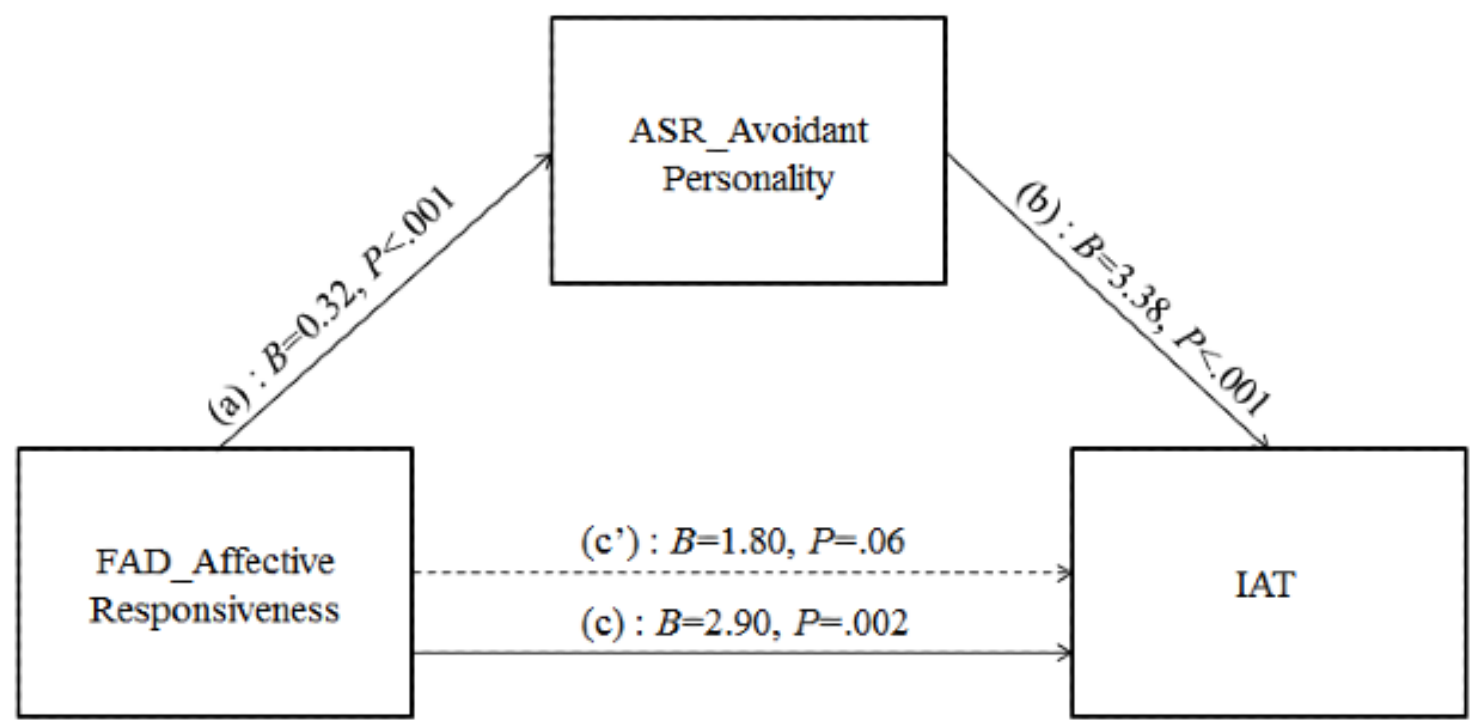

The indirect path via avoided personality traits of young adults was also statistically significant (Table 6).

Finally, results of the multiple mediation analyses showed a significant total indirect effect $(B=1.24 ; 95 \%$ bootstrapped CI
0.28-2.38), implying that the changes in ASR depression, anxiety, and avoidant personality collectively mediated the relationship between FAD AR and IA. However, the specific indirect effects of the 3 mediators were not significant (CI included zero; Table 7). 
Table 7. Multiple mediator model showing total, direct, and indirect effects of affective responsiveness on internet addiction.

\begin{tabular}{|c|c|c|}
\hline \multirow[t]{2}{*}{ Paths } & \multicolumn{2}{|l|}{ Path effect } \\
\hline & $\mathrm{B}(\mathrm{SE})$ & $95 \% \mathrm{CI}$ \\
\hline \multicolumn{3}{|l|}{ Direct path } \\
\hline$\left(\mathrm{A}_{1}\right) \mathrm{AfRes}^{\mathrm{a}} \rightarrow \mathrm{DEP}^{\mathrm{b}}$ & $0.29(0.09)$ & 0.10 to 0.48 \\
\hline$\left(\mathrm{A}_{2}\right) \mathrm{AfRes} \rightarrow \mathrm{ANX}^{\mathrm{c}}$ & $0.15(0.09)$ & -0.04 to 0.34 \\
\hline$\left(\mathrm{A}_{3}\right) \mathrm{AfRes} \rightarrow \mathrm{AvP}^{\mathrm{d}}$ & $0.32(0.09)$ & 0.13 to 0.50 \\
\hline$\left(\mathrm{B}_{1}\right) \mathrm{DEP} \rightarrow \mathrm{IAT}^{\mathrm{e}}$ & $1.86(1.23)$ & -0.59 to 4.32 \\
\hline$\left(\mathrm{B}_{2}\right) \mathrm{ANX} \rightarrow \mathrm{IAT}$ & $0.60(1.16)$ & -1.69 to 2.91 \\
\hline$\left(\mathrm{B}_{3}\right) \mathrm{AvP} \rightarrow \mathrm{IAT}$ & $1.84(1.29)$ & -0.73 to 4.42 \\
\hline (C')AfRes $\rightarrow$ IAT & $1.65(0.95)$ & -0.23 to 3.55 \\
\hline \multicolumn{3}{|l|}{ Indirect path $(A * B)^{\mathbf{f}}$} \\
\hline Total & $1.24(0.53)$ & 0.28 to 2.38 \\
\hline AfRes $\rightarrow$ DEP $\rightarrow$ IAT & $0.55(0.42)$ & -0.17 to 1.48 \\
\hline AfRes $\rightarrow$ ANX $\rightarrow$ IAT & $0.09(0.26)$ & -0.33 to 0.76 \\
\hline AfRes $\rightarrow$ AvP $\rightarrow$ IAT & $0.59(0.47)$ & -0.27 to 1.60 \\
\hline \multicolumn{3}{|l|}{$\operatorname{Total}\left(C=A * B+C^{\prime}\right)^{g}$} \\
\hline AfRes $\rightarrow$ IAT & $2.90(0.94)$ & 1.01 to 4.78 \\
\hline
\end{tabular}

aAfRes: affective responsiveness.

${ }^{b}$ DEP: depression.

${ }^{\mathrm{c}}$ ANX: anxiety.

${ }^{\mathrm{d}}$ AvP: avoidant personality.

${ }^{\mathrm{e}} \mathrm{AAT}$ : internet addiction test.

${ }^{\mathrm{f}}$ Bootstrapped SE values and CI values.

${ }^{\mathrm{g}}$ Proportion mediated by specific indirect effect of AfRes $\rightarrow \mathrm{DEP} \rightarrow \mathrm{IAT}$ is $0.24 \%$, AfRes $\rightarrow$ ANX $\rightarrow$ IAT is $0.05 \%$, and AfRes $\rightarrow$ AvP $\rightarrow \mathrm{IAT}$ is $0.26 \%$.

\section{The Moderator Role Played by Polymorphisms in Young Adults on the Relationship Between Family Functioning and IA}

Finally, we evaluated the possible moderating role played by the genetic polymorphisms in young adults in MAO-A, 5HTTPR, DRD4, and DAT1 genes on the relationship between FAD AR and their scores on IAT. Moderation analyses were conducted using the method outlined by Hayes [91] (Model 1). The results showed that the relationship between FAD AR and IA was moderated by the genotype of the 5-HTTLPR, MAO-A, and DAT1 genes in young adults. The genotype of the DRD4 gene in young adults did not moderate this relationship $(P=.41)$. In particular, high scores on FAD AR were positively associated with high levels of IA, but only in the presence of the L/x genotype of the 5 - HTTLPR gene $(B=.37$; $t_{100}=3.54, P<.001 ; \mathrm{S} / \mathrm{S}: P=.85$; Figure 4).

In addition, the $9 / x$ genotype of the DAT 1 was significantly related with high scores on IAT $\left(B=0.42 ; t_{100}=3.47, P<.001\right.$; 10/10: $P=.56$; Figure 5).

Finally, the results showed a significant interactive effect of the MAOA-H polymorphisms $\quad\left(B=0.44 ; \quad t_{100}=3.55, \quad P<.001\right.$; MAOA-L: $P=.50$; Figure 6 ). 
Figure 4. Moderation of youth's 5-HTTLPR genotype on the relationship between the youth's family functioning and IAT score. 5 - HTTLPR: Serotonin-transporter linked polymorphic region; FAD_AR: Family Assessment Device Affective Responsiveness; IAT: internet addiction test.

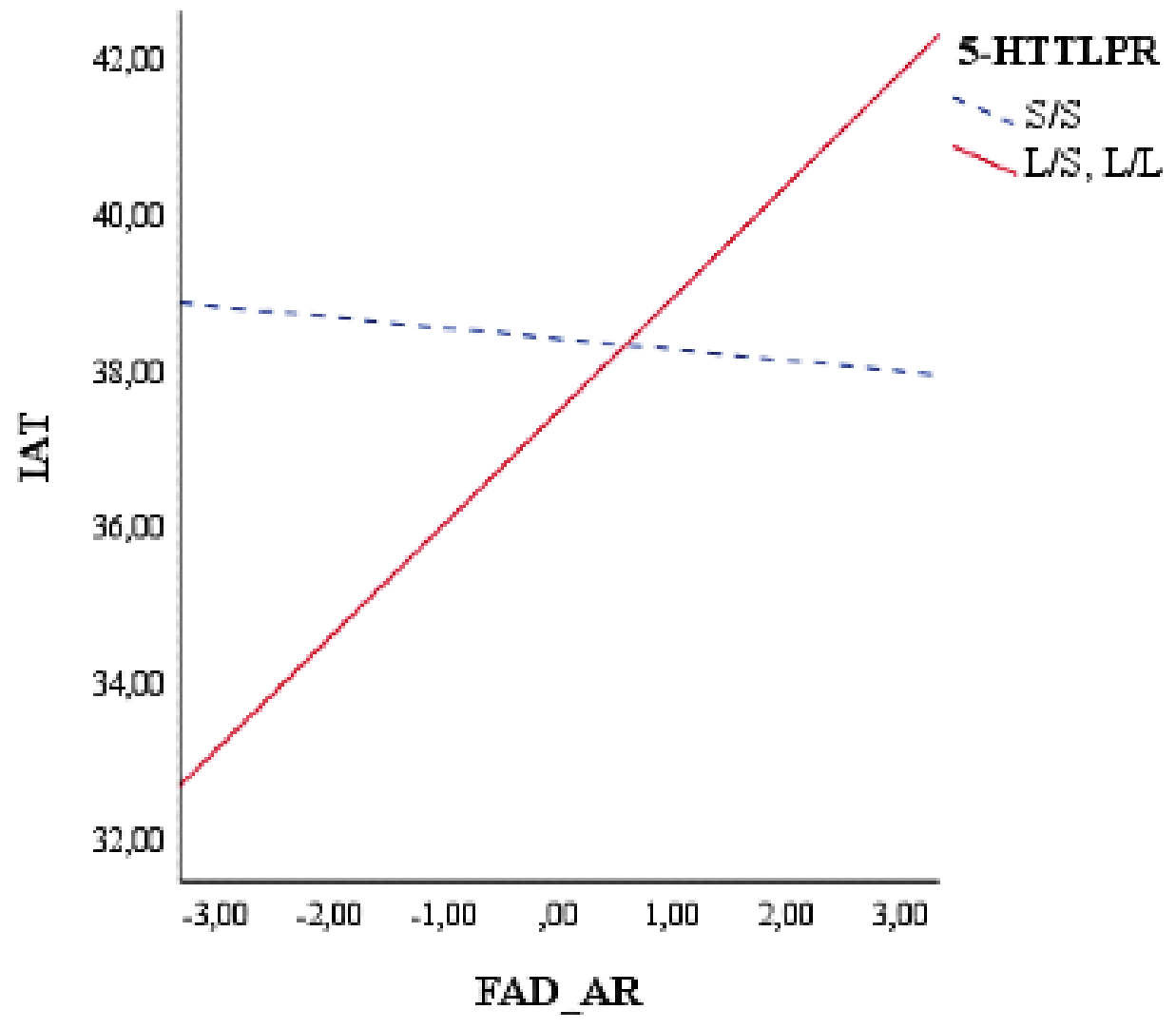

Figure 5. Moderation of youth's DAT1 genotype on the relationship between the youth's family functioning and IAT score. DAT: dopamine active transporter; FAD_AR: Family Assessment Device Affective Responsiveness; IAT: internet addiction test.

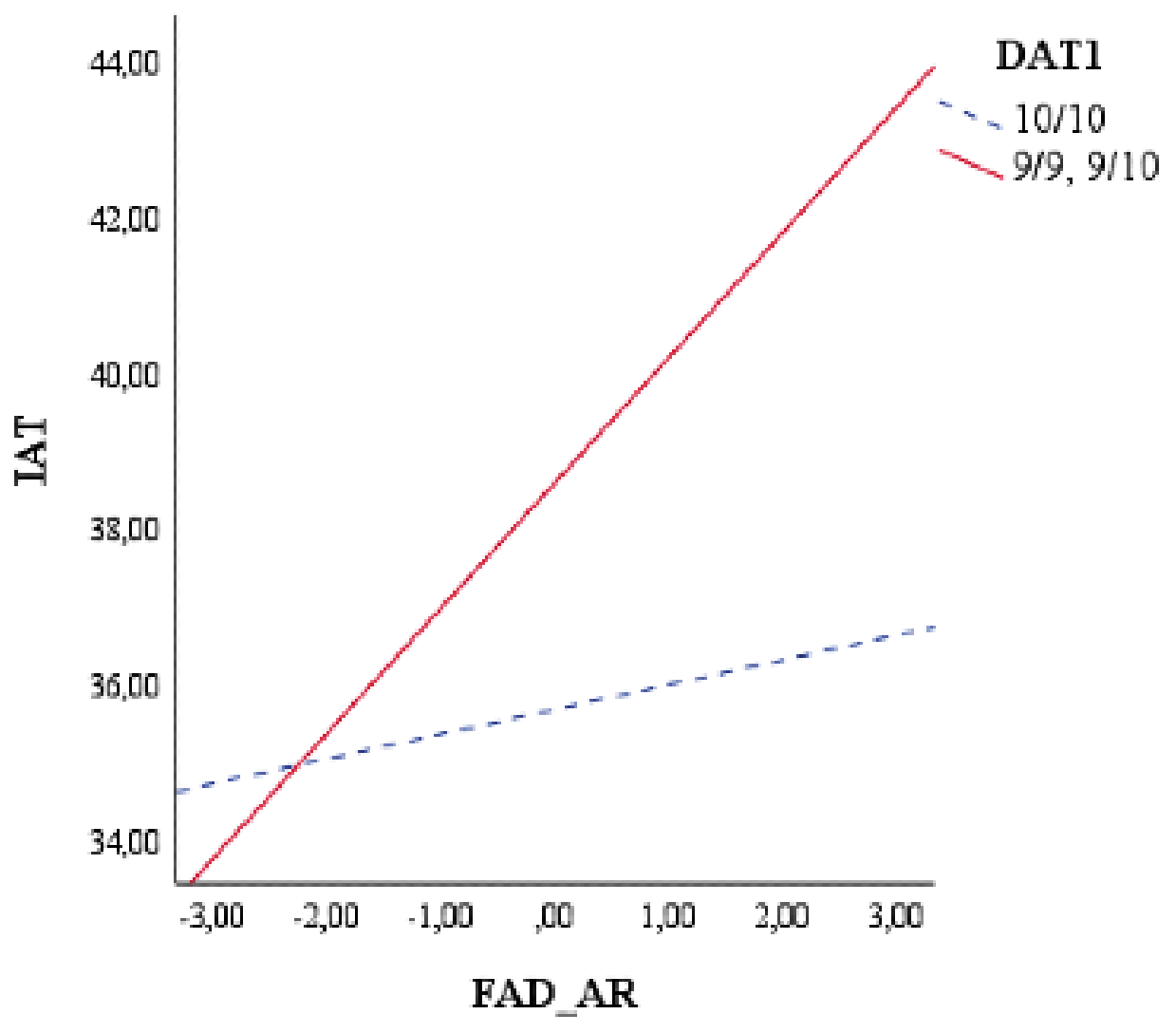


Figure 6. Moderation of the youth's MAO-A genotype on the relationship between youth's family functioning and IAT score. FAD_AR: Family Assessment Device Affective Responsiveness; IAT: internet addiction test; MAO-A: monoamine oxidase A; MAOA-H: MAO-A high-activity variant; MAOA-L: MAO-A low-activity variant.

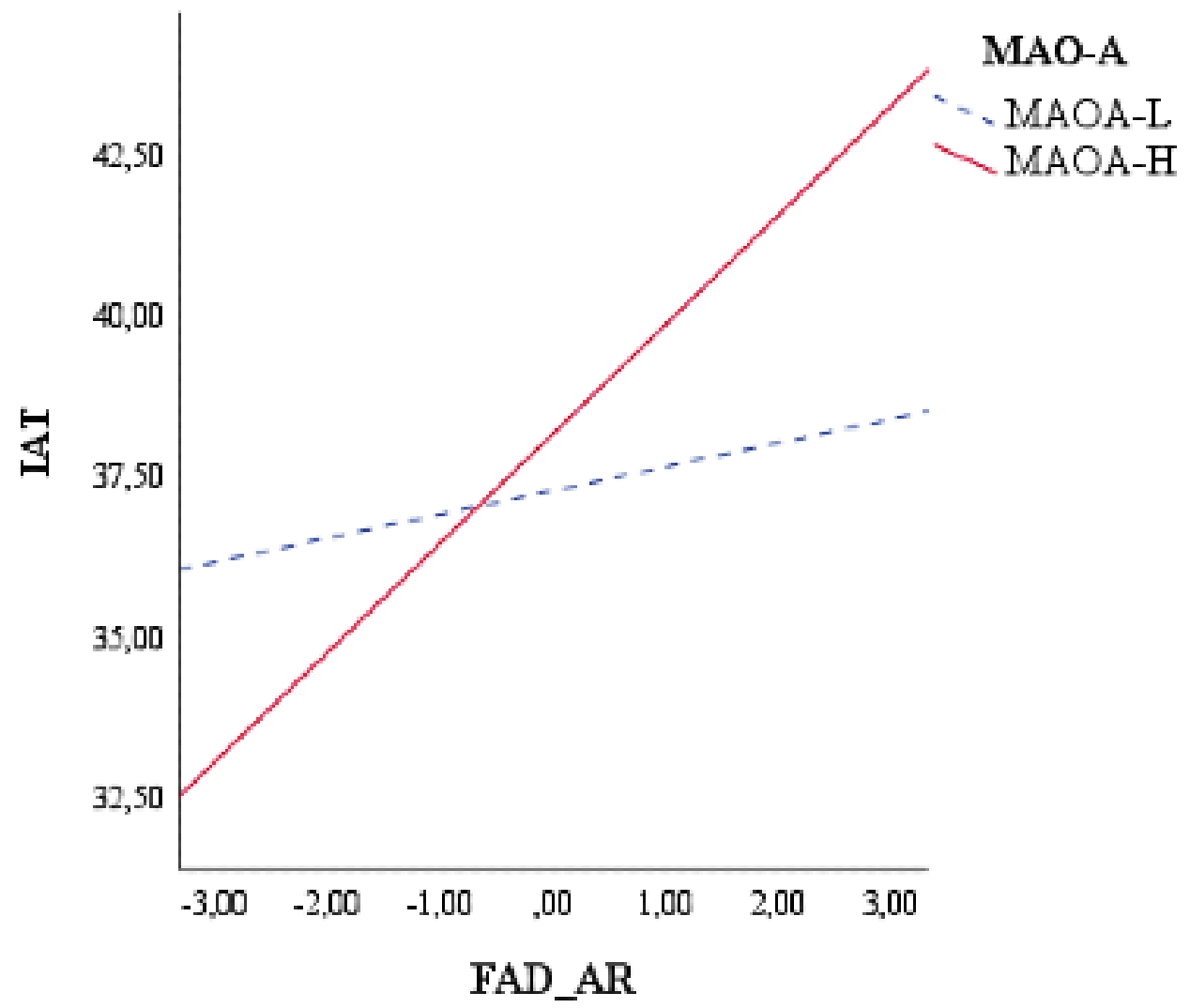

\section{Discussion}

\section{Principal Findings}

This exploratory study aims to investigate the possible biological, psychological, and environmental risk factors associated with IA in a young adult population. On the basis of a biopsychosocial model [71] that considered IA as a result of a mutual influence between individual genotype, psychological profile, and social environment, this study aimed to verify the possible influence of genetic polymorphisms in MAO-A, 5HTTPR, DRD4, and DAT1 genes, considering the role played by the perception of young adults of their family functioning and their psychopathological difficulties. We considered the dopaminergic system (DAT and DRD4 genes), the serotonergic system (5-HTTPLR gene), and the monoamine metabolism pathway (MAO-A gene) for their central role in impulsive behaviors [92-94]. The neurotransmitter systems encoded by these genes are reportedly associated with alcoholism or other addictions [45,72,73,75,95-98]. Moreover, several studies have shown that these genetic polymorphisms contribute to the susceptibility of an individual to environmental influences [99-101] and can act as moderators in associations between the quality of the emotional environment provided by parents and the evolutionary outcomes of young adults [102].

Our first hypothesis was that the genotypes of the 5-HTTPR, DAT1, DRD4, and MAO-A genes differed significantly between the IA and non-IA groups. However, we found no significant association between IA and any genetic polymorphisms, in neither males nor females. Specifically, we found no association between the 5-HTTPR polymorphism and IA. Previous studies [36,41] have reported that the S/S genotype HTTLPR increased the risk of IA. Similarly, our study indicated a higher percentage of the $\mathrm{S} / \mathrm{S}$ genotype in the IA group than in the control group, but there was no significant difference. Moreover, we found no association between the DAT1 genotype and IA. Previous studies have shown that the DAT1 polymorphism is associated with addiction behavior, such as alcoholism [103,104], substance use/abuse [105], and pathological gambling [106]. Some studies $[72,107]$ have suggested that the DAT1 genotype $9 / 9$ is associated with a higher risk for addictive disorders. However, the findings of our study are in line with the findings from studies by other researchers $[74,75]$ that showed no significant association with internet-related addiction. Given these inconsistent findings, further research is needed to clarify whether the DAT1 gene may have an effect on IA. In addition, we found no differences in the DRD4 genotype between the IA and non-IA groups. The DRD4 4/4 polymorphism was shown to be a risk genotype for substance-related disorders [108]. Moreover, it was found to be correlated with decreased risk for inattention $[34,35]$ and, consequently, with increased duration of internet use and a higher risk of IA [36]. However, other studies have underlined a higher risk for addictive disorders associated with the 7-repeat allele of DRD4 [109,110]. In our sample, we found a rarity of the 7-repeat allele $(11 / 104,10.5 \%)$ and, given that previous studies have shown that there was a statistically significant difference in allele distribution of the DRD4 gene between ethnicities [111], further evaluation is necessary for a better understanding of the possible influence of the DRD4 genotype on IA in specific ethnic groups. Finally, 
regarding the MAO-A gene, no significant association was found with IA. However, the literature has reported mixed findings with regard to its genotype that could be considered a higher risk to impulsive behaviors, with some studies showing a significant association with the MAOA low-activity variant $[45,46]$, other studies with the MAOA high-activity variant $[43,44]$, and some other studies that have not found any association [112,113].

Our second hypothesis was that the quality of family functioning perceived by youths and their psychological profiles differed significantly between the IA and non-IA groups. As expected, we found that youths in the IA group reported significantly higher scores (representative of a poorer family functioning) on the subscale of FAD AR and on all psychopathological areas considered (ie, depressive and anxiety symptoms and avoidant personality problems) than their peers in the control group. These findings are consistent with previous studies that have underlined that poor quality of family functioning is one of the main social-environmental risk factors for the onset and maintenance of addictive behaviors among young adult populations [51,52], including IA [49,50,114,115]. Families with poor AR are characterized by difficulties in showing their emotions [84]. In this field, some authors have suggested that, given that feelings are not expressed or tolerated, youths may be more susceptible to cope with their internal stress by themselves [116] and may use the internet excessively as a strategy to cope with negative emotions resulting from the interpersonal relationships with parents [117,118]. Moreover, it has been suggested that young adults who perceive a lack of supportive and intimate relationships with their parents are more likely to search for social support from virtual interactive experiences [119].

With regard to the possible association between IA and psychological difficulties of young adults, our results confirmed that youths of the IA groups showed higher scores on depressive, anxiety, and avoidant personality problems. These findings are in line with previous studies that have shown that young adults affected by IA also reported the presence of more severe psychopathological symptoms than their peers without IA [15], especially in the areas of depression [76,77], anxiety [120], and traits of avoidant personality disorder [70]. The nature of the association between IA and other symptomatic psychopathological areas is controversial. International scientific literature has suggested that psychopathological symptoms may cause or contribute to the onset of IA or, conversely, IA may cause or further exacerbate the course of other psychopathological symptoms $[76,121,122]$. However, recent longitudinal studies $[123,124]$ have produced new evidence on the predictive role of psychological suffering on IA. In this field, some authors have suggested that youths may be at higher risk of developing IA in an attempt to cope with their psychological problems [2]. For example, a youth affected by depression could use the internet as a strategy to escape and cope with feelings of sadness and low self-esteem [122]; excessive use of the internet could be used by young people in an attempt to manage anticipatory anxiety associated with stressful situations and life events [66]; people with avoidant personalities are often socially inhibited and have difficulty communicating with others in face-to-face situations [125], and, thus, they may use web-based communication to seek interpersonal interactions in a way safer and easier for them [126].

Our third hypothesis was that the psychopathological symptoms of young adults might mediate the relationship between FAD $\mathrm{AR}$ and IA. International research has shown that poor quality of family functioning is a significant predictor of the psychopathological symptoms of young adults [2,127], which, in turn, has been reported as a significant predictor of IA $[123,124]$, suggesting a possible mediation role. Our results showed that depressive symptoms and avoidant personality problems of young adults mediated the relationship between FAD AR and IA. Moreover, our results have confirmed previous studies on the significant positive association between the perception of a poor AR of families of young adults to their depressive problems [127,128] and avoidant personality traits [129]. However, anxiety symptoms in youths did not mediate the relationship between FAD AR and IA, although the direct effect on IA was confirmed and is in line with studies by Sepehrian and Lotf [130] and Razieh et al [131]. The results of multiple mediation analyses showed only a significant total indirect effect, indicating that depression and anxiety problems, and avoidant personality traits together significantly explained the association between youth's family AR and IA. However, the specific effects of the 3 mediators were not significant. As evidenced by Hayes [91], these results are only apparently contradictory, and could be as a result of the presence of a correlation between the mediators and the small size of the sample. Although previous research has underlined that psychopathological symptoms of young adults mediate the relationships between environmental risk factors (eg, parental psychopathological symptoms, stressful life events) and IA $[132,133]$, this is the first study to explore the possible role played by family functioning in these processes.

Finally, our fourth hypothesis was that genetic polymorphisms in MAO-A, 5HTTPR, DRD4, and DAT1 genes might moderate the relationship between family functioning and IA. Our results showed that the relationship between FAD AR and IA was moderated by the genotype of the 5 - HTTLPR, DAT1, and MAO-A genes in young adults. In particular, poor AR perceived by youths in their family functioning was associated with a high level of IA, but only in the presence of the L/x genotype of the 5 - HTTLPR gene, the MAOA-H genotype, and the 9/x genotype of the DAT1. These findings are in accordance with recent evidence from geneXenvironment (GxE) interaction studies, which have indicated that not all individuals are susceptible to environmental influences to the same extent [134]. In this regard, the individual genotype (genetic variations) is considered to play a crucial role in moderating individual sensitivity to environmental exposure [79] and to the development of psychopathology related to addiction [48]. In particular, although studies by Lee et al [41] and Sun et al [36] have reported a significant association between IA and the presence of 2 copies of the short allele (S/S) of the 5-HTTLPR, our results are in line with previous studies that have reported an interaction effect between the L allele with stressful life events and a poor quality of family relationship on the onset of psychopathological problems [109,135], including difficulties 
in the area of addiction [136,137]. Moreover, the presence of the MAOA-H genotype gene has been shown to be a significant moderator in the relationship between family environmental exposure and addiction-related problems [138,139], and individuals carrying the 9-repeat allele of the DAT1 gene are at higher risk of alcoholism [140] and other addictive problems $[72,107]$. However, to the best of our knowledge, this is the first study to explore the possible influence of GxE on IA.

\section{Possible Limitations, Strength, and Implications}

This study has some limitations. First, the small sample size and the resulting limited statistical power that should be taken with caution in our preliminary findings, which should be confirmed by further studies with larger samples. Moreover, the cross-sectional nature of the study did not allow testing of causal links between the variables taken into account, which should be explored in subsequent longitudinal studies. Moreover, we used a self-report tool for the assessment of IA of young adults, their family functioning, and psychopathological difficulties. Although these tools have proven their worth in numerous studies, future studies should evaluate these variables through more robust methodologies, such as observational procedures or clinical interviews. Despite these limitations, this exploratory study is the first to focus on the possible moderation of genetic polymorphisms in MAO-A, 5-HTTPR, DRD4, and DAT1 genes on the relationship between the quality of family functioning and IA of young adults, reporting significant GxE effects. Overall, our findings add new evidence with regard to the biological, individual, and social risk factors associated with IA in the young adult population, which can be evaluated for the development of more targeted and effective intervention programs.

\section{Conflicts of Interest}

None declared.

\section{References}

1. Villanti AC, Johnson AL, Ilakkuvan V, Jacobs MA, Graham AL, Rath JM. Social media use and access to digital technology in US young adults in 2016. J Med Internet Res 2017 Jun 7;19(6):e196. [doi: 10.2196/jmir.7303] [Medline: 28592394]

2. Yan W, Li Y, Sui N. The relationship between recent stressful life events, personality traits, perceived family functioning and internet addiction among college students. Stress Health 2014 Feb;30(1):3-11. [doi: 10.1002/smi.2490] [Medline: 23616371]

3. Graham M, Dutton W. In: Graham M, editor. Society and the Internet: How Networks of Information and Communication Are Changing Our Lives. 2th Editions. Oxford: Oxford University Press; 2019.

4. Do TT, Le MD, Van Nguyen T, Tran BX, Le HT, Nguyen HD, et al. Receptiveness and preferences of health-related smartphone applications among Vietnamese youth and young adults. BMC Public Health 2018 Jun 19;18(1):764 [FREE Full text] [doi: 10.1186/s12889-018-5641-0] [Medline: 29921258]

5. Smahel D, Brown BB, Blinka L. Associations between online friendship and internet addiction among adolescents and emerging adults. Dev Psychol 2012;48(2):381-388. [doi: 10.1037/a0027025] [Medline: 22369342]

6. Valkenburg PM, Peter J. Online communication among adolescents: an integrated model of its attraction, opportunities, and risks. J Adolesc Health 2011 Feb;48(2):121-127. [doi: 10.1016/j.jadohealth.2010.08.020] [Medline: 21257109]

7. Ostovar S, Allahyar N, Aminpoor H, Moafian F, Nor MB, Griffiths MD. Internet addiction and its psychosocial risks (depression, anxiety, stress and loneliness) among iranian adolescents and young adults: a structural equation model in a cross-sectional study. Int J Ment Health Addiction 2016 Jan 6;14(3):257-267. [doi: 10.1007/s11469-015-9628-0]

8. Morahan-Martin J. Internet abuse: emerging trends and lingering questions. In: Barak A, editor. Psychological Aspects of Cyberspace: Theory, Research, Applications. Cambridge, United Kingdom: Cambridge University Press; 2008:32-69.

9. Diagnostic and Statistical Manual of Mental Disorders, 5th Edition: DSM-5. Washington, Wash, USA: American Psychiatric Association; 2013.

10. Kuss DJ, Griffiths MD, Pontes HM. Chaos and confusion in DSM-5 diagnosis of internet gaming disorder: issues, concerns, and recommendations for clarity in the field. J Behav Addict 2017 Jun 1;6(2):103-109 [FREE Full text] [doi: 10.1556/2006.5.2016.062] [Medline: 27599673]

11. VandeCreek L, Jackson TL. Internet addiction: symptoms, evaluation and treatment. In: Innovations in Clinical Practice: A Source Book: 17. Sarasota, FL: Professional Resource Press; 1998:237-244.

12. Deryakulu D, Ursavaş ?. Genetic and environmental influences on problematic Internet use: a twin study. Comput Hum Behav 2014 Oct;39:331-338. [doi: 10.1016/j.chb.2014.07.038]

13. Tran BX, Mai HT, Nguyen LH, Nguyen CT, Latkin CA, Zhang MW, et al. Vietnamese validation of the short version of internet addiction test. Addict Behav Rep 2017 Dec;6:45-50 [FREE Full text] [doi: 10.1016/j.abrep.2017.07.001] [Medline: 29450235]

14. Mak K, Lai C, Ko C, Chou C, Kim D, Watanabe H, et al. Psychometric properties of the revised chen internet addiction scale (CIAS-R) in Chinese adolescents. J Abnorm Child Psychol 2014 Oct;42(7):1237-1245. [doi:

10.1007/s10802-014-9851-3] [Medline: 24585392]

15. Ni X, Yan H, Chen S, Liu Z. Factors influencing internet addiction in a sample of freshmen university students in China. Cyberpsychol Behav 2009 Jun;12(3):327-330. [doi: 10.1089/cpb.2008.0321] [Medline: 19445631] 
16. Frangos CC, Frangos CC, Sotiropoulos I. Problematic Internet Use among Greek university students: an ordinal logistic regression with risk factors of negative psychological beliefs, pornographic sites, and online games. Cyberpsychol Behav Soc Netw 2011;14(1-2):51-58. [doi: 10.1089/cyber.2009.0306] [Medline: 21329443]

17. Mak K, Lai C, Watanabe H, Kim D, Bahar N, Ramos M, et al. Epidemiology of internet behaviors and addiction among adolescents in six Asian countries. Cyberpsychol Behav Soc Netw 2014 Nov;17(11):720-728. [doi: 10.1089/cyber.2014.0139] [Medline: 25405785]

18. Stavropoulos V, Alexandraki K, Motti-Stefanidi F. Recognizing internet addiction: prevalence and relationship to academic achievement in adolescents enrolled in urban and rural Greek high schools. J Adolesc 2013 Jun;36(3):565-576. [doi: 10.1016/j.adolescence.2013.03.008] [Medline: 23608781]

19. Hwang JY, Choi J, Gwak AR, Jung D, Choi S, Lee J, et al. Shared psychological characteristics that are linked to aggression between patients with internet addiction and those with alcohol dependence. Ann Gen Psychiatry 2014 Feb 21;13(1):6 [FREE Full text] [doi: 10.1186/1744-859X-13-6] [Medline: 24559036]

20. Cicchetti D, Rogosch FA. Equifinality and multifinality in developmental psychopathology. Dev Psychopathol 2009 Mar 4;8(4):597-600. [doi: 10.1017/s0954579400007318]

21. Cicchetti D, Blender JA. A multiple-levels-of-analysis perspective on resilience: implications for the developing brain, neural plasticity, and preventive interventions. Ann N Y Acad Sci 2006 Dec;1094:248-258. [doi: 10.1196/annals.1376.029] [Medline: 17347356$]$

22. Sameroff A. A unified theory of development: a dialectic integration of nature and nurture. Child Dev 2010;81(1):6-22. [doi: 10.1111/j.1467-8624.2009.01378.x] [Medline: 20331651]

23. Cicchetti D. Developmental Psychopathology, Developmental Neuroscience. New York: John Wiley \& Sons; 2016.

24. Montag C, Reuter M. Molecular genetics, personality,Internet addiction revisited. In: Internet Addiction Neuroscientific Approaches and Therapeutical Interventions. Cham, Switzerland: Springer International Publishing; 2017:141-160.

25. Nieoullon A, Coquerel A. Dopamine: a key regulator to adapt action, emotion, motivation and cognition. Curr Opin Neurol 2003 Dec;16 Suppl 2:S3-S9. [Medline: 15129844]

26. Pierce RC, Kumaresan V. The mesolimbic dopamine system: the final common pathway for the reinforcing effect of drugs of abuse? Neurosci Biobehav Rev 2006;30(2):215-238. [doi: 10.1016/j.neubiorev.2005.04.016] [Medline: 16099045]

27. Nestler EJ, Carlezon WA. The mesolimbic dopamine reward circuit in depression. Biol Psychiatry 2006 Jun 15;59(12):1151-1159. [doi: 10.1016/j.biopsych.2005.09.018] [Medline: 16566899]

28. VanNess SH, Owens MJ, Kilts CD. The variable number of tandem repeats element in DAT1 regulates in vitro dopamine transporter density. BMC Genet 2005 Nov 27;6:55 [FREE Full text] [doi: 10.1186/1471-2156-6-55] [Medline: 16309561]

29. Brand M, Young KS, Laier C, Wölfling K, Potenza MN. Integrating psychological and neurobiological considerations regarding the development and maintenance of specific Internet-use disorders: an interaction of person-affect-cognition-execution (I-PACE) model. Neurosci Biobehav Rev 2016 Dec;71:252-266 [FREE Full text] [doi: 10.1016/j.neubiorev.2016.08.033] [Medline: 27590829]

30. Zhu Y, Zhang H, Tian M. Molecular and functional imaging of internet addiction. Biomed Res Int 2015;2015:378675 [FREE Full text] [doi: 10.1155/2015/378675] [Medline: 25879023]

31. Hou H, Jia S, Hu S, Fan R, Sun W, Sun T, et al. Reduced striatal dopamine transporters in people with Internet addiction disorder. J Biomed Biotechnol 2012;2012:854524 [FREE Full text] [doi: 10.1155/2012/854524] [Medline: 22505818]

32. Roussos P, Giakoumaki SG, Bitsios P. Cognitive and emotional processing in high novelty seeking associated with the L-DRD4 genotype. Neuropsychologia 2009 Jun;47(7):1654-1659. [doi: 10.1016/j.neuropsychologia.2009.02.005] [Medline: $\underline{19397860]}$

33. McGeary J. The DRD4 exon 3 VNTR polymorphism and addiction-related phenotypes: a review. Pharmacol Biochem Behav 2009 Sep;93(3):222-229 [FREE Full text] [doi: 10.1016/j.pbb.2009.03.010] [Medline: 19336242]

34. Kebir O, Joober R. Neuropsychological endophenotypes in attention-deficit/hyperactivity disorder: a review of genetic association studies. Eur Arch Psychiatry Clin Neurosci 2011 Dec;261(8):583-594. [doi: 10.1007/s00406-011-0207-5] [Medline: 21409419]

35. Wu J, Xiao H, Sun H, Zou L, Zhu L. Role of dopamine receptors in ADHD: a systematic meta-analysis. Mol Neurobiol 2012 Jun;45(3):605-620. [doi: 10.1007/s12035-012-8278-5] [Medline: 22610946]

36. Sun C, Spathis R, Sankaranarayanan K, Chan CW, Lum JK. Genetic-linked inattentiveness protects individuals from internet overuse: a genetic study of Internet overuse evaluating hypotheses based on addiction, inattention, novelty-seeking and harm-avoidance. InformingSciJ 2016;19:173-200. [doi: 10.28945/3520]

37. Rotenberg VS. 'Genes of happiness and well being' in the context of search activity concept. Act Nerv Super 2017 Feb 21;55(1-2):1-14. [doi: 10.1007/bf03379593]

38. Mak KK, Kong WY, Mak A, Sharma VK, Ho RC. Polymorphisms of the serotonin transporter gene and post-stroke depression: a meta-analysis. J Neurol Neurosurg Psychiatry 2013 Mar;84(3):322-328. [doi: 10.1136/jnnp-2012-303791] [Medline: 23236014]

39. Nakamura M, Ueno S, Sano A, Tanabe H. The human serotonin transporter gene linked polymorphism (5-HTTLPR) shows ten novel allelic variants. Mol Psychiatry 2000 Jan;5(1):32-38 [FREE Full text] [doi: 10.1038/sj.mp.4000698] [Medline: 10673766] 
40. Gelernter J, Cubells JF, Kidd JR, Pakstis AJ, Kidd KK. Population studies of polymorphisms of the serotonin transporter protein gene. Am J Med Genet 1999 Feb 5;88(1):61-66. [Medline: 10050969]

41. Lee YS, Han DH, Yang KC, Daniels MA, Na C, Kee BS, et al. Depression like characteristics of 5HTTLPR polymorphism and temperament in excessive internet users. J Affect Disord 2008 Jul;109(1-2):165-169. [doi: 10.1016/j.jad.2007.10.020] [Medline: 18045695$]$

42. Sabol SZ, Hu S, Hamer D. A functional polymorphism in the monoamine oxidase a gene promoter. Hum Genet 1998 Sep;103(3):273-279. [doi: 10.1007/s004390050816] [Medline: 9799080]

43. Manuck SB, Flory JD, Ferrell RE, Mann JJ, Muldoon MF. A regulatory polymorphism of the monoamine oxidase-a gene may be associated with variability in aggression, impulsivity, and central nervous system serotonergic responsivity. Psychiatry Res 2000 Jul 24;95(1):9-23. [doi: 10.1016/s0165-1781(00)00162-1] [Medline: 10904119]

44. Fite PJ, Brown S, Hossain W, Manzardo A, Butler MG, Bortolato M. Tobacco and cannabis use in college students are predicted by sex-dimorphic interactions between MAOA genotype and child abuse. CNS Neurosci Ther 2019 Jan;25(1):101-111 [FREE Full text] [doi: 10.1111/cns.13002] [Medline: 29952131]

45. Ibañez A, de Castro IP, Fernandez-Piqueras J, Blanco C, Saiz-Ruiz J. Pathological gambling and DNA polymorphic markers at MAO-A and MAO-B genes. Mol Psychiatry 2000 Jan;5(1):105-109 [FREE Full text] [doi: 10.1038/sj.mp.4000654] [Medline: 10673777]

46. de Castro IP, Ibáñez A, Saiz-Ruiz J, Fernández-Piqueras J. Concurrent positive association between pathological gambling and functional DNA polymorphisms at the MAO-A and the 5-HT transporter genes. Mol Psychiatry 2002;7(9):927-928 [FREE Full text] [doi: 10.1038/sj.mp.4001148] [Medline: 12399942]

47. Ducci F, Goldman D. The genetic basis of addictive disorders. Psychiatr Clin North Am 2012 Jun;35(2):495-519 [FREE Full text] [doi: 10.1016/j.psc.2012.03.010] [Medline: 22640768]

48. Vink JM. Genetics of addiction: future focus on genexenvironment interaction? J Stud Alcohol Drugs 2016 Sep;77(5):684-687. [doi: 10.15288/jsad.2016.77.684] [Medline: 27588524]

49. Kalaitzaki AE, Birtchnell J. The impact of early parenting bonding on young adults' internet addiction, through the mediation effects of negative relating to others and sadness. Addict Behav 2014 Mar;39(3):733-736. [doi: 10.1016/j.addbeh.2013.12.002] [Medline: 24368006]

50. Şenormancı O, Şenormancı G, Güçlü O, Konkan R. Attachment and family functioning in patients with internet addiction. Gen Hosp Psychiatry 2014;36(2):203-207. [doi: 10.1016/j.genhosppsych.2013.10.012] [Medline: 24262601]

51. Velleman RD, Templeton LJ, Copello AG. The role of the family in preventing and intervening with substance use and misuse: a comprehensive review of family interventions, with a focus on young people. Drug Alcohol Rev 2005 Mar;24(2):93-109. [doi: 10.1080/09595230500167478] [Medline: 16076580]

52. Garmendia ML, Alvarado ME, Montenegro M, Pino P. Social support as a protective factor of recurrence after drug addiction treatment. Rev Med Chil 2008 Feb;136(2):169-178 [FREE Full text] [Medline: 18483670 ]

53. Marzilli E, Ballarotto G, Cimino S, Cerniglia L. Incidenti stradali in adolescenza: Il ruolo del supporto genitoriale. Rassegna di Psicologia 2018;34:17-28 [FREE Full text] [doi: 10.4558/8371-02]

54. Yu L, Shek D. Family functioning, positive youth development, and internet addiction in junior secondary school studentstructural equation modeling using AMOS. Int J Disabil Hum Dev 2014;13(2):227-238. [doi: 10.1515/ijdhd-2014-0308]

55. Kabasakal Z. Life satisfaction and family functions as-predictors of problematic Internet use in university students. Comput Hum Behav 2015 Dec;53:294-304. [doi: 10.1016/j.chb.2015.07.019]

56. Li J, Li D, Jia J, Li X, Wang Y, Li Y. Family functioning and internet addiction among adolescent males and females: a moderated mediation analysis. Child Youth Serv Rev 2018 Aug;91:289-297. [doi: 10.1016/j.childyouth.2018.06.032]

57. Cerniglia L, Cimino S, Tafà M, Marzilli E, Ballarotto G, Bracaglia F. Family profiles in eating disorders: family functioning and psychopathology. Psychol Res Behav Manag 2017;10:305-312 [FREE Full text] [doi: 10.2147/PRBM.S145463] [Medline: 29042824]

58. Jozefiak T, Greger HK, Koot HM, Klöckner CA, Wallander JL. The role of family functioning and self-esteem in the quality of life of adolescents referred for psychiatric services: a 3-year follow-up. Qual Life Res 2019 Sep;28(9):2443-2452. [doi: 10.1007/s11136-019-02197-7] [Medline: 31077032]

59. Tafà M, Cerniglia L, Cimino S. Finalmente il coparenting: l'importanza della cogenitorialità per il benessere dei figli. Psic Clin Svil 2019;23(2):201-220. [doi: 10.1449/94339]

60. Moreau A, Laconi S, Delfour M, Chabrol H. Psychopathological profiles of adolescent and young adult problematic Facebook users. Comput Hum Behav 2015 Mar;44:64-69. [doi: 10.1016/j.chb.2014.11.045]

61. Laconi S, Vigouroux M, Lafuente C, Chabrol H. Problematic internet use, psychopathology, personality, defense and coping. Comput Hum Behav 2017 Aug;73:47-54. [doi: 10.1016/j.chb.2017.03.025]

62. Gervasi A, La ML, Lombardo E, Mannino G, Iacolino C, Schimmenti A. Maladaptive personality traits and internet addiction symptoms among young adults: a study based on the alternative DSM-5 model for personality disorders. Clin Neuropsychiatry 2017;14(1).

63. Cerniglia L, Zoratto F, Cimino S, Laviola G, Ammaniti M, Adriani W. Internet addiction in adolescence: neurobiological, psychosocial and clinical issues. Neurosci Biobehav Rev 2017 May;76(Pt A):174-184. [doi: 10.1016/j.neubiorev.2016.12.024] [Medline: 28027952] 
64. Lin Y, Hsiao RC, Liu T, Yen C. Bidirectional relationships of psychiatric symptoms with internet addiction in college students: a prospective study. J Formos Med Assoc 2020 Jun;119(6):1093-1100 [FREE Full text] [doi: 10.1016/j.jfma.2019.10.006] [Medline: 31653577 ]

65. Tran BX, Huong LT, Hinh ND, Nguyen LH, Le BN, Nong VM, et al. A study on the influence of internet addiction and online interpersonal influences on health-related quality of life in young Vietnamese. BMC Public Health 2017 Jan 31;17(1):138 [FREE Full text] [doi: 10.1186/s12889-016-3983-z] [Medline: 28143462]

66. Ho RC, Zhang MW, Tsang TY, Toh AH, Pan F, Lu Y, et al. The association between internet addiction and psychiatric co-morbidity: a meta-analysis. BMC Psychiatry 2014 Jun 20;14:183-192 [FREE Full text] [doi: 10.1186/1471-244X-14-183] [Medline: 24947851]

67. Goel D, Subramanyam A, Kamath R. A study on the prevalence of internet addiction and its association with psychopathology in Indian adolescents. Indian J Psychiatry 2013 Apr;55(2):140-143 [FREE Full text] [doi: 10.4103/0019-5545.111451] [Medline: 23825847]

68. Younes F, Halawi G, Jabbour H, el Osta N, Karam L, Hajj A, et al. Internet addiction and relationships with insomnia, anxiety, depression, stress and self-esteem in university students: a cross-sectional designed study. PLoS One 2016;11(9):e0161126 [FREE Full text] [doi: 10.1371/journal.pone.0161126] [Medline: 27618306]

69. Ko H, Wu JY, Lane H. Personality disorders in female and male college students with internet addiction. J Nerv Ment Dis 2017 Sep;205(9):740-741. [doi: 10.1097/NMD.0000000000000708] [Medline: 28846575]

70. Potembska E, Pawłowska B, Szymańska J. Psychopathological symptoms in individuals at risk of Internet addiction in the context of selected demographic factors. Ann Agric Environ Med 2019 Mar 22;26(1):33-38 [FREE Full text] [doi: 10.26444/aaem/81665] [Medline: $\underline{30922026}$ ]

71. Griffiths M. A 'components' model of addiction within a biopsychosocial framework. J Subst Use 2009 Jul 12;10(4):191-197. [doi: 10.1080/14659890500114359]

72. Brewer AJ, Nielsen DA, Spellicy CJ, Hamon SC, Gingrich J, Thompson-Lake DG, et al. Genetic variation of the dopamine transporter (DAT1) influences the acute subjective responses to cocaine in volunteers with cocaine use disorders. Pharmacogenet Genomics 2015 Jun;25(6):296-304 [FREE Full text] [doi: 10.1097/FPC.0000000000000137] [Medline: 25850966]

73. Chmielowiec J, Chmielowiec K, Suchanecka A, Trybek G, Mroczek B, Małecka I, et al. Associations between the dopamine D4 receptor and DAT1 dopamine transporter genes polymorphisms and personality traits in addicted patients. Int J Environ Res Public Health 2018 Sep 21;15(10) [FREE Full text] [doi: 10.3390/ijerph15102076] [Medline: $\underline{\text { 30248905] }}$

74. Kim K, Ryu E, Chon M, Yeun E, Choi S, Seo J, et al. Internet addiction in Korean adolescents and its relation to depression and suicidal ideation: a questionnaire survey. Int J Nurs Stud 2006 Feb;43(2):185-192. [doi: 10.1016/j.ijnurstu.2005.02.005] [Medline: $\underline{16427966}$ ]

75. Park J, Sung J, Kim D, Kong ID, Hughes TL, Kim N. Genetic association of human corticotropin-releasing hormone receptor 1 (CRHR1) with internet gaming addiction in Korean male adolescents. BMC Psychiatry 2018 Dec 20;18(1):396 [FREE Full text] [doi: 10.1186/s12888-018-1974-6] [Medline: $\underline{\text { 30572854] }}$

76. Hetzel-Riggin MD, Pritchard JR. Predicting problematic Internet use in men and women: the contributions of psychological distress, coping style, and body esteem. Cyberpsychol Behav Soc Netw 2011 Sep;14(9):519-525. [doi: 10.1089/cyber.2010.0314] [Medline: 21342011]

77. Yen C, Chou W, Liu T, Yang P, Hu H. The association of Internet addiction symptoms with anxiety, depression and self-esteem among adolescents with attention-deficit/hyperactivity disorder. Compr Psychiatry 2014 Oct;55(7):1601-1608 [FREE Full text] [doi: 10.1016/j.comppsych.2014.05.025] [Medline: 25015304]

78. Zadra S, Bischof G, Besser B, Bischof A, Meyer C, John U, et al. The association between Internet addiction and personality disorders in a general population-based sample. J Behav Addict 2016 Dec;5(4):691-699 [FREE Full text] [doi: 10.1556/2006.5.2016.086] [Medline: 28005417]

79. Belsky J, Pluess M. Beyond diathesis stress: differential susceptibility to environmental influences. Psychol Bull 2009 Nov;135(6):885-908. [doi: 10.1037/a0017376] [Medline: 19883141]

80. Cimino S, Cerniglia L, Ballarotto G, Marzilli E, Pascale E, D'Addario C, et al. Children's DAT1 polymorphism moderates the relationship between parents' psychological profiles, children's DAT methylation, and their emotional/behavioral functioning in a normative sample. Int J Environ Res Public Health 2019 Jul 18;16(14) [FREE Full text] [doi: 10.3390/ijerph16142567] [Medline: 31323798]

81. Young KS. Internet addiction: the emergence of a new clinical disorder. Cyberpsychol Behav 1998 Jan;1(3):237-244. [doi: $10.1089 / \mathrm{cpb} .1998 .1 .237]$

82. Faraci P, Craparo G, Messina R, Severino S. Internet addiction test (IAT): which is the best factorial solution? J Med Internet Res 2013 Oct 9;15(10):e225 [FREE Full text] [doi: 10.2196/jmir.2935] [Medline: 24184961]

83. Achenbach T, Rescorla L. Manual for the Aseba Adult Forms \& Profiles: For Ages 18-59: Adult Self-Report and Adult Behavior Checklist. USA: Aseba; 2003.

84. Epstein N, Baldwin L, Bishop D. The McMaster family assessment device. J Marital Fam Ther 1983;9(2):171-180. [doi: 10.1111/j.1752-0606.1983.tb01497.x] 
85. Grandi S, Fabbri S, Scortichini S, Bolzani R. Validazione italiana del family assessment device (FAD). Riv Psichiatr 2007;42(2):114-122.

86. Miller I, Epstein N, Bishop D, Keitner G. The McMaster family assessment device: reliability and validity. J Marital Fam Ther 1985;11(4):345-356. [doi: 10.1111/j.1752-0606.1985.tb00028.x]

87. Reist C, Ozdemir V, Wang E, Hashemzadeh M, Mee S, Moyzis R. Novelty seeking and the dopamine D4 receptor gene (DRD4) revisited in Asians: haplotype characterization and relevance of the 2-repeat allele. Am J Med Genet B Neuropsychiatr Genet 2007 Jun 5;144B(4):453-457. [doi: 10.1002/ajmg.b.30473] [Medline: 17474081]

88. Ding Y, Chi H, Grady DL, Morishima A, Kidd JR, Kidd KK, et al. Evidence of positive selection acting at the human dopamine receptor D4 gene locus. Proc Natl Acad Sci U S A 2002 Jan 8;99(1):309-314 [FREE Full text] [doi: 10.1073/pnas.012464099] [Medline: 11756666]

89. Ledwina T, Gnot S. Testing for Hardy-Weinberg equilibrium. Biometrics 1980 Mar;36(1):161. [doi: 10.2307/2530507]

90. Graffelman J, Weir BS. Testing for Hardy-Weinberg equilibrium at biallelic genetic markers on the X chromosome. Heredity (Edinb) 2016 Jun;116(6):558-568 [FREE Full text] [doi: 10.1038/hdy.2016.20] [Medline: 27071844]

91. Hayes A. Introduction to Mediation, Moderation, and Conditional Process Analysis: a Regression-based Approach. 2th Edition. New York: Guilford Publications; 2017.

92. Dalley JW, Roiser JP. Dopamine, serotonin and impulsivity. Neuroscience 2012 Jul 26;215:42-58 [FREE Full text] [doi: 10.1016/j.neuroscience.2012.03.065] [Medline: 22542672]

93. Kolla NJ, Matthews B, Wilson AA, Houle S, Bagby RM, Links P, et al. Lower Monoamine Oxidase-a total distribution volume in impulsive and violent male offenders with antisocial personality disorder and high psychopathic traits: an [(11)C] harmine positron emission tomography study. Neuropsychopharmacology 2015 Oct;40(11):2596-2603 [FREE Full text] [doi: 10.1038/npp.2015.106] [Medline: 26081301]

94. Dellu-Hagedorn F, Rivalan M, Fitoussi A, De Deurwaerdère P. Inter-individual differences in the impulsive/compulsive dimension: deciphering related dopaminergic and serotonergic metabolisms at rest. Philos Trans R Soc Lond B Biol Sci 2018 Apr 19;373(1744) [FREE Full text] [doi: 10.1098/rstb.2017.0154] [Medline: 29483340]

95. Sitte HH, Freissmuth M. Amphetamines, new psychoactive drugs and the monoamine transporter cycle. Trends Pharmacol Sci 2015 Jan;36(1):41-50 [FREE Full text] [doi: 10.1016/j.tips.2014.11.006] [Medline: 25542076]

96. Stewart J. Pathways to relapse: the neurobiology of drug- and stress-induced relapse to drug-taking. J Psychiatry Neurosci 2000 Mar;25(2):125-136 [FREE Full text] [Medline: 10740986]

97. Koda K, Ago Y, Cong Y, Kita Y, Takuma K, Matsuda T. Effects of acute and chronic administration of atomoxetine and methylphenidate on extracellular levels of noradrenaline, dopamine and serotonin in the prefrontal cortex and striatum of mice. J Neurochem 2010 Jul;114(1):259-270 [FREE Full text] [doi: 10.1111/j.1471-4159.2010.06750.x] [Medline: 20403082]

98. Sun-Edelstein C, Tepper SJ, Shapiro RE. Drug-induced serotonin syndrome: a review. Expert Opin Drug Saf 2008 Sep;7(5):587-596. [doi: 10.1517/14740338.7.5.587] [Medline: 18759711 ]

99. Kim-Cohen J, Caspi A, Taylor A, Williams B, Newcombe R, Craig IW, et al. MAOA, maltreatment, and gene-environment interaction predicting children's mental health: new evidence and a meta-analysis. Mol Psychiatry 2006 Oct;11(10):903-913. [doi: 10.1038/sj.mp.4001851] [Medline: 16801953]

100. Beach SR, Brody GH, Todorov AA, Gunter TD, Philibert RA. Methylation at SLC6A4 is linked to family history of child abuse: an examination of the Iowa adoptee sample. Am J Med Genet B Neuropsychiatr Genet 2010 Mar 5;153B(2):710-713 [FREE Full text] [doi: 10.1002/ajmg.b.31028] [Medline: 19739105]

101. Dalton ED, Hammen CL, Najman JM, Brennan PA. Genetic susceptibility to family environment: BDNF Val66met and 5-HTTLPR influence depressive symptoms. J Fam Psychol 2014 Dec;28(6):947-956 [FREE Full text] [doi: 10.1037/fam0000032] [Medline: 25347540]

102. Salvatore JE, Aliev F, Bucholz K, Agrawal A, Hesselbrock V, Hesselbrock M, et al. Polygenic risk for externalizing disorders: gene-by-development and gene-by-environment effects in adolescents and young adults. Clin Psychol Sci 2015 Mar;3(2):189-201 [FREE Full text] [doi: 10.1177/2167702614534211] [Medline: 25821660]

103. Bau CH, Almeida S, Costa FT, Garcia CE, Elias EP, Ponso AC, et al. DRD4 and DAT1 as modifying genes in alcoholism: interaction with novelty seeking on level of alcohol consumption. Mol Psychiatry 2001 Jan;6(1):7-9 [FREE Full text] [doi: 10.1038/sj.mp.4000819] [Medline: 11244477]

104. Grzywacz A, Samochowiec J. [Case-control, family based and screening for DNA sequence variation in the dopamine transporter gene polymorphism DAT 1 in alcohol dependence]. Psychiatr Pol 2008;42(3):443-452. [Medline: 19899571]

105. Stolf AR, Müller D, Schuch JB, Akutagava-Martins GC, Guimaraes LS, Szobot CM, et al. Association between the Intron 8 VNTR polymorphism of the DAT1 gene and crack cocaine addiction. Neuropsychobiology 2017;75(3):141-144. [doi: 10.1159/000485128] [Medline: 29332099]

106. Fagundo AB, Fernández-Aranda F, de la Torre R, Verdejo-García A, Granero R, Penelo E, et al. Dopamine DRD2/ANKK1 Taq1A and DAT1 VNTR polymorphisms are associated with a cognitive flexibility profile in pathological gamblers. J Psychopharmacol 2014 Dec;28(12):1170-1177. [doi: 10.1177/0269881114551079] [Medline: 25237117]

107. Galeeva AR, Gareeva AE, Iur'ev EB, Khusnutdinova EK. [VNTR polymorphisms of the serotonin transporter and dopamine transporter genes in male opiate addicts]. Mol Biol (Mosk) 2002;36(4):593-598. [Medline: 12173460] 
108. Namkoong K, Cheon K, Kim J, Jun J, Lee J. Association study of dopamine D2, D4 receptor gene, GABAA receptor beta subunit gene, serotonin transporter gene polymorphism with children of alcoholics in Korea: a preliminary study. Alcohol 2008 Mar;42(2):77-81. [doi: 10.1016/j.alcohol.2008.01.004] [Medline: 18358985]

109. Laucht M, Becker K, Blomeyer D, Schmidt MH. Novelty seeking involved in mediating the association between the dopamine D4 receptor gene exon III polymorphism and heavy drinking in male adolescents: results from a high-risk community sample. Biol Psychiatry 2007 Jan 1;61(1):87-92. [doi: 10.1016/j.biopsych.2006.05.025] [Medline: 16945348]

110. Mallard TT, Doorley J, Esposito-Smythers CL, McGeary JE. Dopamine D4 receptor VNTR polymorphism associated with greater risk for substance abuse among adolescents with disruptive behavior disorders: preliminary results. Am J Addict 2016 Jan;25(1):56-61. [doi: 10.1111/ajad.12320] [Medline: 26688118]

111. Zhao A, Zhao J, Zhang Y, Tang B, Liu Z, Cheng J. Distribution of genotype and allele frequencies of dopamine D4 receptor gene 48 bp variable number tandem repeat polymorphism in Chinese han population in Hunan. Zhonghua Yi Xue Yi Chuan Xue Za Zhi 2005 Aug;22(4):470-472. [Medline: 16086296]

112. Lu R, Lee J, Ko H, Lin W, Chen K, Shih JC. No association of the MAOA gene with alcoholism among Han Chinese males in Taiwan. Prog Neuropsychopharmacol Biol Psychiatry 2002 Apr;26(3):457-461. [doi: 10.1016/s0278-5846(01)00288-3] [Medline: 11999895$]$

113. Koller G, Bondy B, Preuss UW, Bottlender M, Soyka M. No association between a polymorphism in the promoter region of the MAOA gene with antisocial personality traits in alcoholics. Alcohol 2003;38(1):31-34. [doi: 10.1093/alcalc/agg003] [Medline: 12554604$]$

114. Milani L, Osualdella D, di Blasio P. Quality of interpersonal relationships and problematic Internet use in adolescence. Cyberpsychol Behav 2009 Dec;12(6):681-684. [doi: 10.1089/cpb.2009.0071] [Medline: 19788382]

115. Pace U, Zappulla C, Guzzo G, di Maggio R, Laudani C, Cacioppo M. Internet addiction, temperament, and the moderator role of family emotional involvement. Int J Ment Health Addiction 2014 Jan 7;12(1):52-63. [doi: 10.1007/s11469-013-9468-8]

116. McKay JR, Murphy RT, Rivinus TR, Maisto SA. Family dysfunction and alcohol and drug use in adolescent psychiatric inpatients. J Am Acad Child Adolesc Psychiatry 1991 Nov;30(6):967-972. [doi: 10.1097/00004583-199111000-00015] [Medline: 1757446$]$

117. Richards R, McGee R, Williams SM, Welch D, Hancox RJ. Adolescent screen time and attachment to parents and peers. Arch Pediatr Adolesc Med 2010 Mar;164(3):258-262. [doi: 10.1001/archpediatrics.2009.280] [Medline: 20194259]

118. Ballarotto G, Volpi B, Marzilli E, Tambelli R. Adolescent internet abuse: a study on the role of attachment to parents and peers in a large community sample. Biomed Res Int 2018;2018:5769250 [FREE Full text] [doi: 10.1155/2018/5769250] [Medline: 29707572]

119. Tichon JG, Shapiro M. The process of sharing social support in cyberspace. Cyberpsychol Behav 2003 Apr;6(2):161-170. [doi: $10.1089 / 109493103321640356]$ [Medline: 12804028]

120. Akin A, Iskender M. Internet addiction and depression, anxiety and stress. Int Online J Educ Sci 2011;3(1):138-148.

121. Ko CH, Yen JY, Yen CF, Chen CS, Chen CC. The association between Internet addiction and psychiatric disorder: a review of the literature. Eur Psychiatry 2012 Jan;27(1):1-8. [doi: 10.1016/j.eurpsy.2010.04.011] [Medline: 22153731]

122. Young K. The evolution of Internet addiction disorder. In: Internet Addiction. Cham, Switzerland: Springer International Publishing; 2017:3-18.

123. TAS I. Association between depression, anxiety, stress, social support, resilience and internet addiction: a structural equation modelling. Malays Online J Educ Technol 2019 Jul 1;7(3):1-10. [doi: 10.17220/mojet.2019.03.001]

124. Hsieh K, Hsiao RC, Yang Y, Liu T, Yen C. Predictive effects of sex, age, depression, and problematic behaviors on the incidence and remission of internet addiction in college students: a prospective study. Int J Environ Res Public Health 2018 Dec 14;15(12) [FREE Full text] [doi: 10.3390/ijerph15122861] [Medline: 30558175]

125. Leung L. Stressful life events, motives for Internet use, and social support among digital kids. Cyberpsychol Behav 2007 Apr;10(2):204-214. [doi: 10.1089/cpb.2006.9967] [Medline: 17474837]

126. Esfandiari N, Nouri A, Golparvar M, Yarmohammadian MH. The relationship between social anxiety and online communication among adolescents in the city of Isfahan, Iran. Int J Prev Med 2013 Apr;4(4):390-395 [FREE Full text] [Medline: 23671769]

127. Luebbe AM, Bell DJ. Positive and negative family emotional climate differentially predict youth anxiety and depression via distinct affective pathways. J Abnorm Child Psychol 2014 Aug;42(6):897-911. [doi: 10.1007/s10802-013-9838-5] [Medline: 24356797]

128. Sander JB, McCarty CA. Youth depression in the family context: familial risk factors and models of treatment. Clin Child Fam Psychol Rev 2005 Sep;8(3):203-219 [FREE Full text] [doi: 10.1007/s10567-005-6666-3] [Medline: 16151618]

129. Richards JS, Hartman CA, Jeronimus BF, Ormel J, Reijneveld SA, Veenstra R, et al. Beyond not bad or just okay: social predictors of young adults' wellbeing and functioning (a TRAILS study). Psychol Med 2019 Jul;49(9):1459-1469 [FREE Full text] [doi: 10.1017/S0033291718001976] [Medline: 30229710]

130. Sepehrian F, Lotf J. The rate of prevalence in the Internet addiction and its relationship with anxiety and students? Field of study. Aust j basic appl sci 2011;5(10):1202-1206. 
131. Najafi F, Saravi FK, Navidian A, Raeisi SM. The relationship between internet addiction, loneliness and sleep quality among students of nursing and midwifery faculty. Zahedan J Res Med Sci 2018 Dec 25:- in press(forthcoming). [doi: 10.5812/zjrms.68394]

132. Jang MH, Kim MJ, Choi H. Influences of parental problem drinking on internet addiction among early adolescents: a multiple-mediation analysis. J Addict Nurs 2012 Dec;23(4):258-270. [doi: 10.1097/JAN.0b013e31826f69ac] [Medline: 24622496]

133. Li H, Zou Y, Wang J, Yang X. Role of stressful life events, avoidant coping styles, and neuroticism in online game addiction among college students: a moderated mediation model. Front Psychol 2016;7:1794 [FREE Full text] [doi: 10.3389/fpsyg.2016.01794] [Medline: 27920734]

134. Masten AS, Cicchetti D. Developmental cascades. Dev Psychopathol 2010 Aug;22(3):491-495. [doi: 10.1017/S0954579410000222] [Medline: 20576173]

135. Cervilla JA, Molina E, Rivera M, Torres-González F, Bellón JA, Moreno B, PREDICT Study Core Group. The risk for depression conferred by stressful life events is modified by variation at the serotonin transporter 5HTTLPR genotype: evidence from the Spanish PREDICT-gene cohort. Mol Psychiatry 2007 Aug;12(8):748-755. [doi: 10.1038/sj.mp.4001981] [Medline: 17387319 ]

136. Nilsson KW, Sjöberg RL, Damberg M, Alm PO, Ohrvik J, Leppert J, et al. Role of the serotonin transporter gene and family function in adolescent alcohol consumption. Alcohol Clin Exp Res 2005 Apr;29(4):564-570. [doi:

$\underline{\text { 10.1097/01.alc.0000159112.98941.b0] [Medline: } 15834221 \text { ] }}$

137. Kaufman J, Yang B, Douglas-Palumberi H, Crouse-Artus M, Lipschitz D, Krystal JH, et al. Genetic and environmental predictors of early alcohol use. Biol Psychiatry 2007 Jun 1;61(11):1228-1234. [doi: 10.1016/j.biopsych.2006.06.039] [Medline: 17123474$]$

138. Nilsson KW, Wargelius H, Sjöberg RL, Leppert J, Oreland L. The MAO-A gene, platelet MAO-B activity and psychosocial environment in adolescent female alcohol-related problem behaviour. Drug Alcohol Depend 2008 Jan 11;93(1-2):51-62. [doi: 10.1016/j.drugalcdep.2007.08.022] [Medline: 18029114]

139. Nilsson KW, Comasco E, Åslund C, Nordquist N, Leppert J, Oreland L. MAOA genotype, family relations and sexual abuse in relation to adolescent alcohol consumption. Addict Biol 2011 Apr;16(2):347-355. [doi: 10.1111/j.1369-1600.2010.00238.x] [Medline: 20731636]

140. van der Zwaluw CS, Engels RC, Buitelaar J, Verkes RJ, Franke B, Scholte RH. Polymorphisms in the dopamine transporter gene (SLC6A3/DAT1) and alcohol dependence in humans: a systematic review. Pharmacogenomics 2009 May;10(5):853-866. [doi: 10.2217/pgs.09.24] [Medline: 19450132]

\author{
Abbreviations \\ 5-HT: Serotonin \\ 5-HTTLPR: Serotonin-transporter linked polymorphic region \\ 5-HTTPR: Serotonin-transporter \\ AR: affective responsiveness \\ ASR: Adult Self-Report \\ DA: dopamine \\ DAT: dopamine active transporter \\ DRD4: dopamine D4 receptor \\ FAD: Family Assessment Device \\ GxE: geneXenvironment \\ IA: internet addiction \\ IAT: internet addiction test \\ MAO-A: monoamine oxidase A \\ MAOA-H: MAO-A high-activity variant \\ MAOA-L: MAO-A low-activity variant \\ VNTR: variable number of tandem repetitions
}


Edited by $G$ Eysenbach; submitted 08.12.19; peer-reviewed by R Ho, G Papandonatos, H Koot; comments to author 06.09.20; revised version received 02.10.20; accepted 20.10.20; published 24.12.20

Please cite as:

Cerniglia L, Cimino S, Marzilli E, Pascale E, Tambelli R

Associations Among Internet Addiction, Genetic Polymorphisms, Family Functioning, and Psychopathological Risk: Cross-Sectional Exploratory Study

JMIR Ment Health 2020;7(12):e17341

URL: http://mental.jmir.org/2020/12/e17341/

doi: $\underline{10.2196 / 17341}$

PMID: $\underline{33361057}$

CLuca Cerniglia, Silvia Cimino, Eleonora Marzilli, Esterina Pascale, Renata Tambelli. Originally published in JMIR Mental Health (http://mental.jmir.org), 24.12.2020. This is an open-access article distributed under the terms of the Creative Commons Attribution License (https://creativecommons.org/licenses/by/4.0/), which permits unrestricted use, distribution, and reproduction in any medium, provided the original work, first published in JMIR Mental Health, is properly cited. The complete bibliographic information, a link to the original publication on http://mental.jmir.org/, as well as this copyright and license information must be included. 